Research Article

\title{
Nonlinear Vibration of a Functionally Graded Nanobeam Based on the Nonlocal Strain Gradient Theory considering Thickness Effect
}

\author{
Dang-Van Hieu $\mathbb{D}^{1},{ }^{1}$ The-Hung Duong ${ }^{(D)},{ }^{1}$ and Gia-Phi Bui ${ }^{2}$ \\ ${ }^{1}$ Thai Nguyen University of Technology, Thainguyen, Vietnam \\ ${ }^{2}$ University of Transport Technology, Hanoi, Vietnam \\ Correspondence should be addressed to The-Hung Duong; hungtd@tnut.edu.vn
}

Received 12 December 2019; Revised 11 June 2020; Accepted 19 June 2020; Published 9 July 2020

Academic Editor: Jinyang Xu

Copyright (c) 2020 Dang-Van Hieu et al. This is an open access article distributed under the Creative Commons Attribution License, which permits unrestricted use, distribution, and reproduction in any medium, provided the original work is properly cited.

\begin{abstract}
In this work, a nonlocal strain gradient beam model considering the thickness effect is developed to study the nonlinear vibration response of a functionally graded nanobeam. The governing equation of the functionally graded nanobeam is derived by using the Euler-Bernoulli beam theory with von Kármán's nonlinear strain-gradient relationship and the Hamilton principle. The expression of the nonlinear frequency for the functionally graded nanobeam with pinned-pinned boundary conditions is obtained with the help of Galerkin technique and the Hamiltonian approach. The obtained results show that the effect of thickness is very important for the size-dependent vibration response of the functionally graded nanobeam; the nonlinear vibration response of the nanobeam depends not only on the material length scale parameter and nonlocal parameter but also on the slenderness ratio. Effects of the slenderness ratio and the power-law index on the vibration response of the functionally graded nanobeam are also investigated and discussed. The numerical results show that the nonlocal parameter reduces the nonlinear frequency of the functionally graded nanobeam, while the material length scale parameter increases the nonlinear frequency of the functionally graded nanobeam. The slenderness ratio leads to an increase in the nonlinear frequency of the functionally graded nanobeam, while the power-law index leads to a decrease in the nonlinear frequency of the functionally graded nanobeam.
\end{abstract}

\section{Introduction}

Because of wide applications in areas such as micro-/nanoelectromechanical systems (MEMs/NEMs), biosensors, micro-/nanosensors, and nanoactuators, the behaviors of micro-/nanostructures have attracted attention of many scientists. Experiment observations showed that the sizedependent influences of the micro-/nanostructures are very important and cannot be ignored $[1,2]$. Some higher-order elasticity theories that can capture the size-dependent effects on the behaviors of the micro-/nanostructures have been developed. The nonlocal elasticity theory introduced by Eringen $[3,4]$ states that the stress at a point is considered as a function of strains at all points in the continuum body. This theory is widely used to analyze responses of the structures at the nanoscale. Based on the nonlocal elasticity theory, a number of works on the static and dynamic behaviors of beams have been published [5-11]. The vibration and stability problems of the carbon nanotubes based on the nonlocal elasticity theory have also attracted attention of many authors [12-20]. It can be seen that the nonlocal elasticity theory has been employed very effectively for modeling the nanostructures; however, research results show that only the stiffness-softening effect is observed by using this theory.

The strain gradient theory (SGT) [21-25] states that the total stress should consider some additional strain gradient terms by modeling small-scaled materials as atoms with the higher-order deformation mechanism instead of collection of points. By modifying the SGT, Yang et al. [26] proposed the modified couple stress theory (MCST); this theory considers the strain energy density as a function of both the 
strain tensor conjugated with the stress tensor and the curvature tensor conjugated with the couple stress tensor. In 2003, with the similar approach as Yang et al. [26], Lam et al. [27] introduced the modified strain gradient theory (MSGT) with three material length scale parameters. Other than the nonlocal elasticity theory, the SGT, MCST, and MSGT play stiffness-hardening effect on responses of the micro-/ nanostructures. Based on the SGT and MCST, many works related to the static and dynamic responses of beams were carried out [28-37].

It can be seen that the nonlocal elasticity theory and the SGT describe size-dependent nano-/micromechanical characteristics of materials in two different manners. In 2015, Lim et al. [38] combined the nonlocal elasticity theory and the strain gradient theory into a generalized higher-order elasticity theory called the nonlocal strain gradient theory (NSGT). Effects of the two length scales including the material length scale parameter and the nonlocal parameter on the mechanical and physical behaviors of size-dependent structures are described in the NSGT. The NSGT can be considered the most generalized theory of the higher-order elasticity theories that can describe the behaviors of size-dependent structures. Many articles related to the static and dynamic behaviors of micro-/nanostructures have been published based on the NSGT. Based on the NSGT, Li and Hu [39] investigated the buckling problem of size-dependent nonlinear beams. A size-dependent sinusoidal shear deformation beam model was developed by Lu et al. [40] for analyzing the free vibration response of nanobeams. The NSGT was used by Li et al. [41] to examine size-dependent effects on critical flow velocity of fluid-conveying microtubes. Bahaadini et al. [42] investigated free vibration and instability behaviors of a nanotube conveying nanoflow based on the NSGT and the Timoshenko beam theory. The nonlinear dynamical behavior of a fluid-conveying viscoelastic microtube was studied by Ghayesh and Farokhi using the NSGT and the Euler-Bernoulli beam theory [43]. The NSGT was employed by Arefi et al. to investigate the size-dependent bending response of a sandwich porous nanoplate integrated with piezomagnetic face-sheets [44] and the magneto-electroelastic vibration response of a porous functionally graded core sandwich nanoplate with piezomagnetic face-sheets and resting on an elastic foundation [45].

First discovered by scientists in Japan in 1984, the functionally graded materials (FGMs) have some advantages over the traditional composite materials. FGMs are increasingly being used extensively in the construction of structures that work in environments requiring high temperature resistance, corrosion resistance, improved stress spreading, and inferior stress intensity factors [46]. There is a large number of works analyzing mechanical behaviors of functionally graded (FG) structures. Ansari et al. [47] investigated free vibration characteristics of FG microbeams based on the strain gradient Timoshenko beam theory. Based on the MCST and the Euler-Bernoulli beam theory, Arbind and Reddy [48] studied nonlinear vibration responses of FG microbeams. Nonlinear free vibration and postbuckling behaviors of FG beams resting on the nonlinear elastic layer were examined by Fallah and Aghdam
[49]. Bending and vibration responses of the nonlocal FG beams were reported by Thai and Vo [50] using various higher-order shear deformation beam theories. The NSGT [38] was also used very effectively to analyze the vibration response of FG beams. Simsek [51] investigated the nonlinear free vibration of a FG nanobeam based on the NSGT and the Euler-Bernoulli beam theory. Based on the NSGT, size-dependent nonlinear Euler-Bernoulli and Timoshenko beam models were developed by $\mathrm{Li}$ et al. [52] to study bending and free vibration characteristics of FG beams. The NSGT was employed by Allam and Radwan [53] to investigate bending, buckling, and vibration responses of viscoelastic FG curved nanobeam resting on an elastic foundation. Surface effects on nonlinear vibration behavior of an electrostatic FG nanoresonator were investigated by Esfahani et al. [54] using the NSGT and the Euler-Bernoulli beam theory. The effect of the neutral surface on the electroelastic analysis of FG piezoelectric plate resting on the Winkler-Pasternak foundation was investigated by Arefi et al. [55] in the framework of the two-variable sinusoidal shear deformation theory. Arefi et al. [56] studied the free vibration of a sandwich nanoplate including the FG core and piezoelectric face-sheets by using the two-variable sinusoidal shear deformation theory.

The NSGT [38] is being used more and more extensively in examining the size-dependent effects on the mechanical behaviors of micro-/nanostructures. However, in previous publications, the size-dependent effects are often assumed to be neglected in the thickness direction. Recently in 2018, Li et al. [57] showed that the size-dependent effect of thickness on the mechanical behaviors of structures is very important. In [57], the authors developed a nonlocal strain gradient beam model incorporating the thickness to investigate the buckling problem of nanobeams. When considering the effect of thickness, the stiffness-softening and stiffnesshardening effects depend not only on the ratio of the stressgradient parameter to the strain gradient parameter but also on the geometric feature (slenderness ratio) [57]. Nonlinear free vibration response of homogeneous nanobeams was investigated by Chen et al. [58] using the NSGT incorporating the thickness effect.

According to authors' knowledge, nonlinear vibration behavior of the FG nanobeam based on the NSGT and considering the influence of the thickness is not yet announced. Thus, the nonlinear vibration response of the FG nanobeam based on the NSGT incorporating the thickness effect will be investigated in this paper. The governing equation of the FG nanobeam is derived by using the Euler-Bernoulli beam theory with von Kármán’s nonlinear strain-gradient relationship and the Hamilton principle. With the help of Galerkin technique and the Hamiltonian approach, the expression of nonlinear frequency for the FG nanobeam with pinned-pinned boundary conditions is obtained in a closed form. The results show that the effect of thickness is very important to the size-dependent vibration response of the FG nanobeam; the nonlinear vibration response of the FG nanobeam depends not only on the material length scale parameter and nonlocal parameter but also on the slenderness ratio. Effects of the slenderness ratio 
and the power-law index on the vibration response of the FG nanobeam are also investigated and discussed in this paper.

\section{Model and Formulation}

2.1. Modeling of the Functionally Graded Nanobeam. A FG nanobeam of length $L$, width $d$, and height $h$ is considered as in Figure 1. The material properties of the nanobeam vary as a function of the thickness coordinate. Based on the rule of mixture, the material properties $P$ of the nanobeam including Young's modulus $E$ and the material density $\rho$ are expressed as

$$
P=P_{U} V_{U}+P_{L} V_{L}
$$

where $P_{U}$ and $P_{L}$ are the material properties at the upper and lower surfaces of the nanobeam, respectively, and $V_{U}$ and $V_{L}$ are the volume fractions at the upper and lower surfaces of the nanobeam, respectively. The volume fractions $V_{U}$ and $V_{L}$ are supposed as

$$
\begin{aligned}
& V_{U}=\left(\frac{z_{\mathrm{ms}}}{h}+\frac{1}{2}\right)^{k}, \\
& V_{L}=1-\left(\frac{z_{\mathrm{ms}}}{h}+\frac{1}{2}\right)^{k} .
\end{aligned}
$$

Here, $k$ is the power-law index which prescribes the material variation and $z_{\mathrm{ms}}$ is the thickness coordinate from the geometry neutral surface. From equations (1)-(3), the material properties can be obtained as

$$
P\left(z_{\mathrm{ms}}\right)=\left(P_{U}-P_{L}\right)\left(\frac{z_{\mathrm{ms}}}{h}+\frac{1}{2}\right)^{k}+P_{L} .
$$

Because the material properties of the FG nanobeam are not symmetric about its geometry neutral surface, the geometry and the physical neutral surface of the FG nanobeam are not the same. The position of the physical neutral surface can be determined as [51]

$$
c=\frac{\int_{-h / 2}^{h / 2} E\left(z_{\mathrm{ms}}\right) z_{\mathrm{ms}} \mathrm{d} z_{\mathrm{ms}}}{\int_{-h / 2}^{h / 2} E\left(z_{\mathrm{ms}}\right) \mathrm{d} z_{\mathrm{ms}}} .
$$

For a rectangular FG nanobeam, the position of the physical neutral surface can be expressed as [52]

$$
c=\frac{\left(E_{U}-E_{L}\right) h k}{2(2+k)\left(E_{U}+k E_{L}\right)} .
$$

As in Figure 1, the thickness coordinate from the physical neutral surface, $z$, is

$$
z=z_{\mathrm{ms}}-c .
$$

Thus, in the new coordinate system, by substituting equation (7) into equation (4), the material properties of the nanobeam can be expressed as

$$
P(z)=\left(P_{U}-P_{L}\right)\left(\frac{z+c}{h}+\frac{1}{2}\right)^{k}+P_{L} .
$$

Based on equation (8), Young's modulus $E$ and the material density $\rho$ of the FG nanobeam are determined as

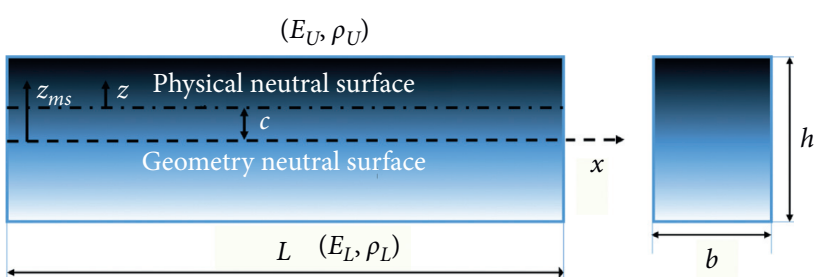

Figure 1: Modeling of a FG nanobeam.

$$
\begin{aligned}
& E(z)=\left(E_{U}-E_{L}\right)\left(\frac{z+c}{h}+\frac{1}{2}\right)^{k}+E_{L}, \\
& \rho(z)=\left(\rho_{U}-\rho_{L}\right)\left(\frac{z+c}{h}+\frac{1}{2}\right)^{k}+\rho_{L} .
\end{aligned}
$$

It can be seen from equations (9) and (10) that $E=E_{U}$, $\rho=\rho_{U}$ when $z=h / 2-c$ and $E=E_{L}, \rho=\rho_{L}$ when $z=$ $-h / 2-c$.

2.2. The Nonlocal Strain Gradient Theory. Based on the NSGT proposed by Lim et al. [38], the total stress of the beam is defined as

$$
t_{x x}=\sigma_{x x}-\nabla \sigma_{x x}^{(1)}
$$

where $\nabla=\partial / \partial x$ is the differential operator and $\sigma_{x x}$ and $\sigma_{x x}^{(1)}$ are the nonlocal stress and the higher-order nonlocal stress, respectively, which are expressed as

$$
\begin{aligned}
& \sigma_{x x}=\int_{0}^{L} E \alpha_{0}\left(x, x^{\prime}, e_{0} a\right) \varepsilon_{x x}^{\prime}\left(x^{\prime}\right) \mathrm{d} x^{\prime}, \\
& \sigma_{x x}^{(1)}=l_{m}^{2} \int_{0}^{L} E \alpha_{1}\left(x, x^{\prime}, e_{1} a\right) \varepsilon_{x x, x}^{\prime}\left(x^{\prime}\right) \mathrm{d} x^{\prime} .
\end{aligned}
$$

In equations (12) and (13), $E$ is Young's modulus, $l_{m}$ is the material length scale parameter or the strain gradient length scale parameter, $\varepsilon_{x x}$ is the classical strain, $\varepsilon_{x x, x}$ is the strain gradient, $\alpha_{0}$ and $\alpha_{1}$ are the two nonlocal kernel functions satisfying the conditions given by Eringen [3, 4], and $e_{0} a$ and $e_{1} a$ are the two nonlocal parameters.

For the one-dimensional problem, by applying the linear operators $L_{i}=1-\left(e_{i} a\right)^{2} \nabla^{2}$ for $i=0,1$ into equation (11), the general nonlocal strain gradient constitutive equation can be obtained as [38]

$$
\begin{aligned}
{\left[1-\left(e_{0} a\right)^{2} \nabla^{2}\right]\left[1-\left(e_{1} a\right)^{2} \nabla^{2}\right] t_{x x}=} & E\left[1-\left(e_{1} a\right)^{2} \nabla^{2}\right] \varepsilon_{x x} \\
& -E l_{m}^{2}\left[1-\left(e_{0} a\right)^{2} \nabla^{2}\right] \nabla^{2} \varepsilon_{x x},
\end{aligned}
$$

in which $\nabla^{2}=\partial^{2} / \partial x^{2}$ is the Laplace operator. When considering $e_{0}=e_{1}=e$, equation (14) can be reduced as

$$
\left[1-(e a)^{2} \nabla^{2}\right] t_{x x}=\left(E-E l_{m}^{2} \nabla^{2}\right) \varepsilon_{x x} .
$$

By letting $l_{m}=0$, equation (15) becomes

$$
\left[1-(e a)^{2} \nabla^{2}\right] t_{x x}=E \varepsilon_{x x}
$$

which is the nonlocal constitutive equation for the nonlocal elasticity theory $[3,4]$. 
Also, on the contrary, the strain gradient constitutive equation for the strain gradient theory [25] can be obtained by letting $e a=0$ :

$$
t_{x x}=\left(E-E l_{m}^{2} \nabla^{2}\right) \varepsilon_{x x} .
$$

2.3. The Governing Equation for a FG Nanobeam considering Thickness Effect. The displacements of the FG nanobeam based on the Euler-Bernoulli beam theory take the form

$$
\begin{aligned}
& u_{1}(x, z, t)=u(x, t)-z \frac{\partial w(x, t)}{\partial x}, \\
& u_{2}(x, z, t)=0, \\
& u_{3}(x, z, t)=w(x, t) .
\end{aligned}
$$

According to von Kármán's geometrical relationship, the nonzero strain of the nanobeam is given as

$$
\varepsilon_{x x}=\frac{\partial u}{\partial x}+\frac{1}{2}\left(\frac{\partial w}{\partial x}\right)^{2}-z \frac{\partial^{2} w}{\partial x^{2}}
$$

where $u$ and $w$ are the axial and transverse displacements of any point on the physical neutral surface, respectively. We can see from equation (19) that the strain of the nanobeam depends not only on $x$ but also on $z$; thus, Li et al. [57] proposed that Laplace operator $\nabla^{2}=\partial^{2} / \partial x^{2}$ in Section 2.2 should be replaced by $\nabla^{2}=\partial^{2} / \partial x^{2}+\partial^{2} / \partial z^{2}$. According to the suggestion by Li et al. [57], the size-dependent effect of thickness will be considered for analyzing the mechanical behavior of the FG nanobeam. By considering the thickness effect, the total stress of the FG nanobeam can be expressed as [57]

$$
t_{x x}=\sigma_{x x}+\sigma_{x x x}^{(1)}+\sigma_{x x z}^{(1)},
$$

where $\sigma_{x x}$ is the nonlocal stress and $\sigma_{x x x}^{(1)}$ and $\sigma_{x x z}^{(1)}$ are the higher-order nonlocal stresses. The virtual strain energy can be given as [57]

$$
\delta U=\int_{V}\left(\sigma_{x x} \delta \varepsilon_{x x}+\sigma_{x x x}^{(1)} \delta \varepsilon_{x x, x}+\sigma_{x x z}^{(1)} \delta \varepsilon_{x x, z}\right) \mathrm{d} V .
$$

Substituting the expression of the strain in equation (19) into equation (21), we get

$$
\begin{aligned}
\delta U= & \int_{V} \sigma_{x x} \delta\left(\frac{\partial u}{\partial x}+\frac{1}{2}\left(\frac{\partial w}{\partial x}\right)^{2}-z \frac{\partial^{2} w}{\partial x^{2}}\right) \mathrm{d} V \\
& +\int_{V} \sigma_{x x x}^{(1)} \delta\left(\frac{\partial^{2} u}{\partial x^{2}}+\frac{\partial w}{\partial x} \frac{\partial^{2} w}{\partial x^{2}}-z \frac{\partial^{3} w}{\partial x^{3}}\right) \mathrm{d} V \\
& +\int_{V} \sigma_{x x z}^{(1)} \delta\left(-\frac{\partial^{2} w}{\partial x^{2}}\right) \mathrm{d} V \\
= & \int_{0}^{L} \int_{A} \sigma_{x x} \delta\left(\frac{\partial u}{\partial x}+\frac{1}{2}\left(\frac{\partial w}{\partial x}\right)^{2}-z \frac{\partial^{2} w}{\partial x^{2}}\right) \mathrm{d} A \mathrm{~d} x \\
& +\int_{0}^{L} \int_{A} \sigma_{x x x}^{(1)} \delta\left(\frac{\partial^{2} u}{\partial x^{2}}+\frac{\partial w}{\partial x} \frac{\partial^{2} w}{\partial x^{2}}-z \frac{\partial^{3} w}{\partial x^{3}}\right) \mathrm{d} A \mathrm{~d} x \\
& +\int_{0}^{L} \int_{A} \sigma_{x x z}^{(1)} \delta\left(-\frac{\partial^{2} w}{\partial x^{2}}\right) \mathrm{d} A \mathrm{~d} x .
\end{aligned}
$$




$$
\begin{aligned}
\left(1-(e a)^{2} \nabla^{2}\right) N_{z} & =-l_{m}^{2} A_{x x} \frac{\partial^{2} w}{\partial x^{2}}, \\
\left(1-(e a)^{2} \nabla^{2}\right) M & =-D_{x x} \frac{\partial^{2} w}{\partial x^{2}} \\
\left(1-(e a)^{2} \nabla^{2}\right) M_{x} & =-l_{m}^{2} D_{x x} \frac{\partial^{3} w}{\partial x^{3}},
\end{aligned}
$$

where

$$
\begin{aligned}
& A_{x x}=\int_{A} E(z) \mathrm{d} A=b \int_{-h / 2-c}^{h / 2-c}\left[\left(E_{U}-E_{L}\right)\left(\frac{z+c}{h}+\frac{1}{2}\right)^{k}+E_{L}\right] \mathrm{d} z, \\
& D_{x x}=\int_{A} E(z) z^{2} \mathrm{~d} A=b \int_{-h / 2-c}^{h / 2-c}\left[\left(E_{U}-E_{L}\right)\left(\frac{z+c}{h}+\frac{1}{2}\right)^{k}+E_{L}\right] z^{2} \mathrm{~d} z .
\end{aligned}
$$

The virtual kinetic energy of the FG nanobeam taking into account both axial and transverse motions is given as

$$
\begin{aligned}
\delta K_{e}= & \int_{0}^{L} \int_{A} \rho(z) \frac{\partial u_{1}}{\partial t}\left(\frac{\partial u_{1}}{\partial t}\right) \mathrm{d} A \mathrm{~d} x \\
& +\int_{0}^{L} \int_{A} \rho(z) \frac{\partial u_{3}}{\partial t} \delta\left(\frac{\partial u_{3}}{\partial t}\right) \mathrm{d} A \mathrm{~d} x \\
\approx & \int_{0}^{L} m_{0} \frac{\partial u}{\partial t} \delta\left(\frac{\partial u}{\partial t}\right) \mathrm{d} x+\int_{0}^{L} m_{0} \frac{\partial w}{\partial t} \delta\left(\frac{\partial w}{\partial t}\right) \mathrm{d} x \\
& +\int_{0}^{L} m_{2} \frac{\partial^{2} w}{\partial t \partial x} \delta\left(\frac{\partial^{2} w}{\partial t \partial x}\right) \mathrm{d} x
\end{aligned}
$$

where $m_{0}$ and $m_{2}$ are the mass moments of inertia which are defined as

$$
\begin{aligned}
& m_{0}=\int_{A} \rho(z) \mathrm{d} A=b \int_{-h / 2-c}^{h / 2-c}\left[\left(\rho_{U}-\rho_{L}\right)\left(\frac{z+c}{h}+\frac{1}{2}\right)^{k}+\rho_{L}\right] \mathrm{d} z, \\
& m_{2}=\int_{A} \rho(z) z^{2} \mathrm{~d} A=b \int_{-h / 2-c}^{h / 2-c}\left[\left(\rho_{U}-\rho_{L}\right)\left(\frac{z+c}{h}+\frac{1}{2}\right)^{k}+\rho_{L}\right] z^{2} \mathrm{~d} z .
\end{aligned}
$$

The virtual work caused by the distributed transverse force, $q(x, t)$, is

$$
\delta W=\int_{0}^{L}(q \delta w) \mathrm{d} x
$$

Hamilton's principle states that

$$
\int_{0}^{t}\left(\delta K_{e}-\delta U+\delta W\right) \mathrm{d} t=0
$$

Substituting equations (24), (31), and (33) into equation (34) and after some mathematical manipulations, we can obtain the following equations:

$$
\begin{gathered}
\delta u:-\frac{\partial N}{\partial x}+\frac{\partial^{2} N_{x}}{\partial x^{2}}=-m_{0} \frac{\partial^{2} u}{\partial t^{2}} \\
\delta w:-\frac{\partial}{\partial x}\left(N \frac{\partial w}{\partial x}\right)-\frac{\partial}{\partial x}\left(N_{x} \frac{\partial^{2} w}{\partial x^{2}}\right)+\frac{\partial^{2}}{\partial x^{2}}\left(N_{x} \frac{\partial w}{\partial x}\right) \\
-\frac{\partial^{2} M}{\partial x^{2}}+\frac{\partial^{3} M_{x}}{\partial x^{3}}-\frac{\partial^{2} N_{z}}{\partial x^{2}}+q=-m_{0} \frac{\partial^{2} w}{\partial t^{2}}+m_{2} \frac{\partial^{4} w}{\partial t^{2} \partial x^{2}}
\end{gathered}
$$

The boundary conditions at $x=0$ and $x=L$ can be expressed as

$$
\begin{aligned}
\delta u: N-\frac{\partial N_{x}}{\partial x} & =0 \\
\text { or } u & =0 \\
\delta\left(\frac{\partial u}{\partial x}\right): N_{x} & =0 \\
\operatorname{or} \frac{\partial u}{\partial x} & =0
\end{aligned}
$$

$\delta w: N \frac{\partial w}{\partial x}+N_{x} \frac{\partial^{2} w}{\partial x^{2}}-\frac{\partial^{2} M_{x}}{\partial x^{2}}+\frac{\partial M}{\partial x}+\frac{\partial N_{z}}{\partial x}-\frac{\partial}{\partial x}\left(N_{x} \frac{\partial w}{\partial x}\right)=0$

or $w=0$,

$$
\begin{array}{r}
\delta\left(\frac{\partial w}{\partial x}\right): N_{x} \frac{\partial w}{\partial x}+\frac{\partial M_{x}}{\partial x}-M-N_{z}=0 \\
\text { or } \frac{\partial w}{\partial x}=0
\end{array}
$$

$$
\begin{aligned}
\delta\left(\frac{\partial^{2} w}{\partial x^{2}}\right): M_{x} & =0 \\
\text { or } \frac{\partial^{2} w}{\partial x^{2}} & =0 .
\end{aligned}
$$

From equations (36) and (37), we get

$$
\begin{aligned}
& -\left(N-\frac{\partial N_{x}}{\partial x}\right) \frac{\partial^{2} w}{\partial x^{2}}+\frac{\partial^{2}}{\partial x^{2}}\left(-M+\frac{\partial M_{x}}{\partial x}-N_{z}\right)+q \\
& =-m_{0} \frac{\partial^{2} w}{\partial t^{2}}+m_{2} \frac{\partial^{4} w}{\partial t^{2} \partial x^{2}} .
\end{aligned}
$$

Let

$$
N^{*}=N-\frac{\partial N_{x}}{\partial x}
$$

The expression for $N^{*}$ can be extended from the result for the homogeneous beam obtained by Chen et al. [58] as follows:

$$
N^{*}=\frac{A_{x x}}{2 L} \int_{0}^{L}\left(\frac{\partial w}{\partial x}\right)^{2} \mathrm{~d} x
$$

From equations (27)-(29), we obtain 


$$
\begin{aligned}
\left(1-(e a)^{2} \nabla^{2}\right)\left(\frac{\partial M_{x}}{\partial x}-M-N_{z}\right)= & -l_{m}^{2} D_{x x} \frac{\partial^{4} w}{\partial x^{4}}+D_{x x} \frac{\partial^{2} w}{\partial x^{2}} \\
& +l_{m}^{2} A_{x x} \frac{\partial^{2} w}{\partial x^{2}}
\end{aligned}
$$

Now, substituting equations (44) and (45) into equation (42), the governing equation of motion for the FG nanobeam can be obtained as follows:

$$
\begin{aligned}
\left(D_{x x} \frac{\partial^{4} w}{\partial x^{4}}-l_{m}^{2} D_{x x} \frac{\partial^{6} w}{\partial x^{6}}+l_{m}^{2} A_{x x} \frac{\partial^{4} w}{\partial x^{4}}\right) \\
-\frac{A_{x x}}{2 L} \int_{0}^{L}\left(\frac{\partial w}{\partial x}\right)^{2} \mathrm{~d} x\left[\frac{\partial^{2} w}{\partial x^{2}}-(e a)^{2} \frac{\partial^{4} w}{\partial x^{4}}\right] \\
+\left[1-(e a)^{2} \frac{\partial^{2}}{\partial x^{2}}\right]\left(m_{0} \frac{\partial^{2} w}{\partial t^{2}}-m_{2} \frac{\partial^{4} w}{\partial t^{2} \partial x^{2}}\right) \\
=-\left[1-(e a)^{2} \frac{\partial^{2}}{\partial x^{2}}\right] q .
\end{aligned}
$$

In the case of pinned-pinned boundary conditions, the classical and nonclassical boundary conditions for the nanobeam at $x=0$ and $x=L$ are [57]

$$
w=\frac{\partial^{2} w}{\partial x^{2}}=\frac{\partial^{4} w}{\partial x^{4}}=0 .
$$

Equation (46) is the governing equation of motion for the FG nonlocal strain gradient nanobeam considering the thickness effect. For the homogeneous nanobeam, i.e., $D_{x x}=\mathrm{EI}, A_{x x}=\mathrm{EA}, m_{0}=\rho A$, and the second mass moment of inertia $m_{2}=\rho I$ is ignored, equation (46) becomes the governing equation of motion for the homogeneous nonlocal strain gradient nanobeam considering the thickness effect developed by Chen et al. [58]:

$$
\begin{gathered}
\left(\mathrm{EI} \frac{\partial^{4} w}{\partial x^{4}}-l_{m}^{2} \mathrm{EI} \frac{\partial^{6} w}{\partial x^{6}}+l_{m}^{2} \mathrm{EA} \frac{\partial^{4} w}{\partial x^{4}}\right)-\frac{\mathrm{EA}}{2 L} \int_{0}^{L}\left(\frac{\partial w}{\partial x}\right)^{2} d x \\
\cdot\left[\frac{\partial^{2} w}{\partial x^{2}}-(e a)^{2} \frac{\partial^{4} w}{\partial x^{4}}\right] \\
+\rho A\left[\frac{\partial^{2} w}{\partial t^{2}}-(e a)^{2} \frac{\partial^{4} w}{\partial t^{2} \partial x^{2}}\right]=-\left[1-(e a)^{2} \frac{\partial^{2}}{\partial x^{2}}\right] q .
\end{gathered}
$$

When the thickness effect is not taken into account, equation (46) is reduced to the model of the FG Euler-Bernoulli beam developed by $\mathrm{Li}$ and $\mathrm{Hu}$ [52]:

$$
\begin{aligned}
\left(D_{x x} \frac{\partial^{4} w}{\partial x^{4}}-l_{m}^{2} D_{x x} \frac{\partial^{6} w}{\partial x^{6}}\right)-\frac{A_{x x}}{2 L} \int_{0}^{L}\left(\frac{\partial w}{\partial x}\right)^{2} \mathrm{~d} x \\
\cdot\left[\frac{\partial^{2} w}{\partial x^{2}}-(e a)^{2} \frac{\partial^{4} w}{\partial x^{4}}\right]+\left[1-(e a)^{2} \frac{\partial^{2}}{\partial x^{2}}\right] \\
\cdot\left(m_{0} \frac{\partial^{2} w}{\partial t^{2}}-m_{2} \frac{\partial^{4} w}{\partial t^{2} \partial x^{2}}\right) \\
=-\left[1-(e a)^{2} \frac{\partial^{2}}{\partial x^{2}}\right] q .
\end{aligned}
$$

For convenience, the following dimensionless variables are introduced:

$$
\begin{aligned}
& \bar{x}=\frac{x}{L}, \\
& \bar{w}=\frac{w}{L}, \\
& \bar{t}=t \sqrt{\frac{E_{L} h^{2}}{\rho_{L} L^{4}}}, \\
& \beta=\frac{l_{m}}{L}, \\
& \alpha=\frac{e a}{L}, \\
& \gamma=\frac{L}{h}, \\
& \bar{z}=\frac{z}{h}, \\
& \bar{c}=\frac{c}{h} .
\end{aligned}
$$

For the purpose of studying the free vibration, the transverse distributed load is ignored, and using equation (50), the equation of motion for the FG nanobeam can be rewritten in a dimensionless form as follows:

$$
\begin{aligned}
& \left(\bar{D}_{x x} \frac{\partial^{4} \bar{w}}{\partial \bar{x}^{4}}-\beta^{2} \bar{D}_{x x} \frac{\partial^{6} \bar{w}}{\partial \bar{x}^{6}}+\beta^{2} \gamma^{2} \bar{A}_{x x} \frac{\partial^{4} \bar{w}}{\partial \bar{x}^{4}}\right) \\
& -\frac{\bar{A}_{x x}}{2} \gamma^{2} \int_{0}^{1}\left(\frac{\partial \bar{w}}{\partial \bar{x}}\right)^{2} d \bar{x}\left[\frac{\partial^{2} \bar{w}}{\partial \bar{x}^{2}}-\alpha^{2} \frac{\partial^{4} \bar{w}}{\partial \bar{x}^{4}}\right] \\
& +\bar{m}_{0}\left(\frac{\partial^{2} \bar{w}}{\partial \bar{t}^{2}}-\alpha^{2} \frac{\partial^{4} \bar{w}}{\partial \bar{t}^{2} \partial \bar{x}^{2}}\right)-\bar{m}_{2} \frac{1}{\gamma^{2}}\left(\frac{\partial^{4} \bar{w}}{\partial \bar{t}^{2} \partial \bar{x}^{2}}-\alpha^{2} \frac{\partial^{6} \bar{w}}{\partial \bar{t}^{2} \partial \bar{x}^{4}}\right)=0
\end{aligned}
$$

where 


$$
\begin{aligned}
& \bar{A}_{x x}=b \int_{-1 / 2-\bar{c}}^{1 / 2-\bar{c}}\left[\left(\frac{E_{U}}{E_{L}}-1\right)\left(\bar{z}+\bar{c}+\frac{1}{2}\right)^{k}+1\right] \mathrm{d} \bar{z}, \\
& \bar{D}_{x x}=b \int_{-1 / 2-\bar{c}}^{1 / 2-\bar{c}}\left[\left(\frac{E_{U}}{E_{L}}-1\right)\left(\bar{z}+\bar{c}+\frac{1}{2}\right)^{k}+1\right] \bar{z}^{2} \mathrm{~d} \bar{z}, \\
& \bar{m}_{0}=b \int_{-1 / 2-\bar{c}}^{1 / 2-\bar{c}}\left[\left(\frac{\rho_{U}}{\rho_{L}}-1\right)\left(\bar{z}+\bar{c}+\frac{1}{2}\right)^{k}+1\right] \mathrm{d} \bar{z}, \\
& \bar{m}_{2}=b \int_{-1 / 2-\bar{c}}^{1 / 2-\bar{c}}\left[\left(\frac{\rho_{U}}{\rho_{L}}-1\right)\left(\bar{z}+\bar{c}+\frac{1}{2}\right)^{k}+1\right] \bar{z}^{2} \mathrm{~d} \bar{z} .
\end{aligned}
$$

The classical and nonclassical boundary conditions at $\bar{x}=0$ and $\bar{x}=1$ become

$$
\bar{w}=\frac{\partial^{2} \bar{w}}{\partial \bar{x}^{2}}=\frac{\partial^{4} \bar{w}}{\partial \bar{x}^{4}}=0 .
$$

Now, we will apply the Galerkin method to convert partial differential equation (51) into the ordinary differential one. By this method, the displacement function is assumed to have the following form:

$$
\bar{w}(\bar{x}, t \bar{t})=V(\bar{t}) \phi(\bar{x})
$$

in which $V(\bar{t})$ is an unknown time-dependent function and needs to be determined, and $\phi(\bar{x})$ is a shape function that satisfies the boundary conditions of the nanobeam. For the pinned-pinned nanobeam, the shape function can be chosen as

$$
\phi(\bar{x})=\sin (\pi \bar{x}) .
$$

Applying the Galerkin method, equation (51) is reduced to the following ordinary differential equation:

$$
\ddot{V}(\bar{t})+\gamma_{1} V(\bar{t})+\gamma_{2} V^{3}(\bar{t})=0,
$$

where coefficients $\gamma_{1}$ and $\gamma_{2}$ are given as

$$
\begin{aligned}
& \gamma_{1}=\frac{\bar{D}_{x x} \int_{0}^{1} \phi^{(4)} \phi \mathrm{d} \bar{x}-\beta^{2} \bar{D}_{x x} \int_{0}^{1} \phi^{(6)} \phi d \bar{x}+\beta^{2} \gamma^{2} \bar{A}_{x x} \int_{0}^{1} \phi^{(4)} \phi \mathrm{d} \bar{x}}{\bar{m}_{0}\left(\int_{0}^{1} \phi^{2} \mathrm{~d} \bar{x}-\alpha^{2} \int_{0}^{1} \phi^{\prime \prime} \phi \mathrm{d} \bar{x}\right)-\bar{m}_{2}\left(1 / \gamma^{2}\right)\left(\int_{0}^{1} \phi^{\prime \prime} \phi \mathrm{d} \bar{x}-\alpha^{2} \int_{0}^{1} \phi^{(4)} \phi \mathrm{d} \bar{x}\right)}, \\
& \gamma_{2}=\frac{-\gamma^{2}\left(\bar{A}_{x x} / 2\right) \int_{0}^{1}\left(\phi^{\prime}\right)^{2} \mathrm{~d} \bar{x}\left[\int_{0}^{1} \phi^{\prime \prime} \phi \mathrm{d} \bar{x}-\alpha^{2} \int_{0}^{1} \phi^{(4)} \phi \mathrm{d} \bar{x}\right]}{\bar{m}_{0}\left(\int_{0}^{1} \phi^{2} \mathrm{~d} \bar{x}-\alpha^{2} \int_{0}^{1} \phi^{\prime \prime} \phi \mathrm{d} \bar{x}\right)-\bar{m}_{2}\left(1 / \gamma^{2}\right)\left(\int_{0}^{1} \phi^{\prime \prime} \phi \mathrm{d} \bar{x}-\alpha^{2} \int_{0}^{1} \phi^{(4)} \phi \mathrm{d} \bar{x}\right)},
\end{aligned}
$$

where $\phi^{(6)}=\mathrm{d}^{6} \phi / \mathrm{d} \bar{x}^{6}, \phi^{(4)}=\mathrm{d}^{4} \phi / \mathrm{d} \bar{x}^{4}, \phi^{\prime \prime}=\mathrm{d}^{2} \phi / \mathrm{d} \bar{x}^{2}$, and $\phi^{\prime}=\mathrm{d} \phi / \mathrm{d} \bar{x}$.

\section{Solution Procedure}

In this section, we will find the approximate solution of equation (56). As can be seen that equation (56) is a Duffing oscillator, there are many techniques to find the approximate solution of this equation. The Hamiltonian approach proposed by He [59] can be applied to find the approximate solution of equation (56). First, we assume that equation (56) satisfies the following initial conditions:

$$
\begin{aligned}
& V(0)=V_{0}, \\
& \dot{V}(0)=0,
\end{aligned}
$$

where $V_{0}=\bar{w}(0.5)$ is the dimensionless maximum vibration amplitude of the nanobeam.

According to the Hamiltonian approach [59], the Hamiltonian of equation (31) can be constructed as

$$
H=\frac{1}{2} \dot{V}^{2}(\bar{t})+\frac{1}{2} \gamma_{1} V^{2}(\bar{t})+\frac{1}{4} \gamma_{2} V^{4}(\bar{t})=\text { const. }
$$
we get:

$$
\bar{H}(V)=\left[\int_{0}^{T / 4} \frac{1}{2} \dot{V}^{2}(\bar{t})+\frac{1}{2} \gamma_{1} V^{2}(\bar{t})+\frac{1}{4} \gamma_{2} V^{4}(\bar{t})\right] \mathrm{d} \bar{t},
$$

where $T$ is the period of oscillation.

Assume that the solution of equation (56) can be given as

$$
V(\bar{t})=V_{0} \cos (\omega \bar{t}) .
$$

Here, $\omega$ is the frequency of oscillation. Substituting equation (62) into equation (61), we get

$$
\begin{aligned}
\bar{H} & =\left[\int_{0}^{T / 4} \frac{1}{2} V_{0}^{2} \omega^{2} \sin ^{2}(\omega \bar{t})+\frac{1}{2} \gamma_{1} V_{0}^{2} \cos ^{2}(\omega \bar{t})+\frac{1}{4} \gamma_{2} V_{0}^{4} \cos ^{4}(\omega \bar{t})\right] \mathrm{d} \bar{t} \\
& =\frac{1}{2} V_{0}^{2} \omega \frac{\pi}{4}+\frac{1}{\omega} \frac{\gamma_{1}}{2} V_{0}^{2} \frac{\pi}{4}+\frac{1}{\omega} \frac{\gamma_{2}}{4} V_{0}^{4} \frac{3 \pi}{16} .
\end{aligned}
$$

The approximate frequency of oscillation can be obtained by setting [59]

$$
\frac{\partial}{\partial V_{0}}\left[\frac{\partial \bar{H}}{\partial(1 / \omega)}\right]=0 .
$$

From equations (63) and (64), the amplitude-frequency relationship of equation (56) can be obtained as follows:

$$
\omega_{\mathrm{NL}}=\sqrt{\gamma_{1}+\frac{3}{4} \gamma_{2} V_{0}^{2}} .
$$


TABLE 1: The material properties of aluminum and alumina [50].

\begin{tabular}{lcc}
\hline Materials & Young's modulus $E(\mathrm{GPa})$ & $\begin{array}{c}\text { Mass density } \rho \\
\left(\mathrm{kg} / \mathrm{m}^{3}\right)\end{array}$ \\
\hline Aluminum (metal) & $E_{U}=380$ & $\rho_{U}=3960$ \\
Alumina (ceramic) & $E_{L}=70$ & $\rho_{L}=2702$ \\
\hline
\end{tabular}

TABLE 2: The variation of the physical neutral surface to the power-law index.

\begin{tabular}{llccccccc}
\hline$k$ & 0 & 0.1 & 0.5 & 1 & 2 & 3 & 4 & 5 \\
\hline$c / h$ & 0 & 0.0191 & 0.0747 & 0.1148 & 0.1490 & 0.1576 & 0.1566 & 0.1517 \\
\hline
\end{tabular}

TABLE 3: The first nondimensional natural frequencies of FG beams.

\begin{tabular}{lcccc}
\hline$k$ & \multicolumn{2}{c}{$L / h=5$} & \multicolumn{2}{c}{$L / h=20$} \\
Thai and Vo [50] & Present & Thai and Vo [50] & 5.4777 \\
\hline 0 & 5.3953 & 5.3953 & 4.4777 & 4.6641 \\
0.5 & 4.5931 & 4.5932 & 4.6641 & 4.2163 \\
1 & 4.1484 & 4.1485 & 3.8472 & 3.8472 \\
2 & 3.7793 & 3.7796 & 3.6628 & 3.6628 \\
5 & 3.5949 & 3.5952 & 3.5547 & 3.5547 \\
10 & 3.4921 & 3.4923 & & Present \\
\hline
\end{tabular}

Substituting the shape function in equation (55) into the expressions of coefficients $\gamma_{1}$ and $\gamma_{2}$ in equations (57) and (58) and calculating the integrals, the amplitude-frequency relationship for the FG nanobeam with pinned-pinned boundary conditions is obtained as

$$
\omega_{\mathrm{NL}}=\sqrt{\frac{\bar{D}_{x x} \pi^{4}\left(1+\beta^{2} \pi^{2}\right)+\bar{A}_{x x} \beta^{2} \gamma^{2} \pi^{4}}{\left(1+\alpha^{2} \pi^{2}\right)\left(\bar{m}_{0}+\left(\left(\bar{m}_{2} \pi^{2}\right) / \gamma^{2}\right)\right)+\frac{3}{4} \bar{A}_{x x} \gamma^{2} \pi^{4} / \bar{m}_{0}+\left(\left(\bar{m}_{2} \pi^{2}\right) / \gamma^{2}\right) V_{0}^{2}}}
$$

Equation (66) is the nondimensional nonlinear frequency of the FG nanobeam considering the thickness effect. The nondimensional linear frequency of the FG nanobeam can be obtained from equation (66) by letting $V_{0}=0$ as follows:

$$
\omega_{L}=\sqrt{\frac{\bar{D}_{x x} \pi^{4}\left(1+\beta^{2} \pi^{2}\right)+\bar{A}_{x x} \beta^{2} \gamma^{2} \pi^{4}}{\left(1+\alpha^{2} \pi^{2}\right)\left(\bar{m}_{0}+\left(\left(\bar{m}_{2} \pi^{2}\right) / \gamma^{2}\right)\right)}}
$$

\section{Results and Discussion}

In this section, various numerical examples are presented and discussed to verify the accuracy of the present results in predicting the free nonlinear vibration responses of the FG nanobeams. A nanobeam composed of aluminum (metal) and alumina (ceramic) is considered. The material properties of aluminum and alumina are shown in Table 1 [50]. The variation of the physical neutral surface to the powerlaw index $k$ is presented in Table 2 .
Table 3 shows the first nondimensional natural frequencies (linear frequencies) of the FG classical beams for different values of the power-law index $k$ and the slenderness ratio $(L / h)$. It can be observed that the results obtained for the Euler-Bernoulli beam theory are in good agreement with the results given by Thai and Vo [50].

From equation (67), we can see that the nondimensional natural frequency of the FG nanobeam depends not only on the material length scale parameter $\left(\beta=l_{m} / L\right)$ and the nonlocal parameter $(\alpha=e a / L)$ but also on the slenderness ratio $(\gamma=L / h)$. The material length scale parameter increases the natural frequency of the FG nanobeam, while the nonlocal parameter decreases the natural frequency of the FG nanobeam. For two cases of $\alpha=0.01, \beta=0.01$ and $\alpha=0.02, \beta=0.03$, Tables 4 and 5 show the first nondimensional natural frequencies of the FG nanobeams for different values of the power-law index $k$ and the slenderness ratio $(L / h)$, respectively. We can see that the slenderness ratio $L / h$ has a great influence on the natural frequency of the FG nanobeam, and the slenderness ratio increases the natural frequency of the FG nanobeam. On the contrary, the 
TABLE 4: The first nondimensional natural frequencies of FG nanobeams for $\alpha=0.01$ and $\beta=0.01$.

\begin{tabular}{|c|c|c|c|}
\hline$L / h$ & $k$ & $\omega_{L}$ without thickness effect & $\omega_{L}$ with thickness effect \\
\hline \multirow{6}{*}{5} & 0 & 5.3953 & 5.4756 \\
\hline & 0.5 & 4.5932 & 4.6699 \\
\hline & 1 & 4.1485 & 4.2217 \\
\hline & 2 & 3.7796 & 3.8454 \\
\hline & 5 & 3.5952 & 3.6473 \\
\hline & 10 & 3.4923 & 3.5372 \\
\hline \multirow{6}{*}{20} & 0 & 5.4777 & 6.6029 \\
\hline & 0.5 & 4.6641 & 5.7853 \\
\hline & 1 & 4.2163 & 5.2825 \\
\hline & 2 & 3.8472 & 4.8088 \\
\hline & 5 & 3.6628 & 4.4361 \\
\hline & 10 & 3.5547 & 4.2262 \\
\hline
\end{tabular}

TABLE 5: The first nondimensional natural frequencies of FG nanobeams for $\alpha=0.02$ and $\beta=0.03$.

\begin{tabular}{|c|c|c|c|}
\hline$L / h$ & $k$ & $\omega_{L}$ without thickness effect & $\omega_{L}$ with thickness effect \\
\hline \multirow{6}{*}{5} & 0 & 5.3821 & 5.6947 \\
\hline & 0.5 & 4.5820 & 4.8798 \\
\hline & 1 & 4.1383 & 4.4224 \\
\hline & 2 & 3.7703 & 4.0259 \\
\hline & 5 & 3.5864 & 3.7893 \\
\hline & 10 & 3.4838 & 3.6590 \\
\hline \multirow{6}{*}{20} & 0 & 5.4643 & 9.3254 \\
\hline & 0.5 & 4.6527 & 8.2550 \\
\hline & 1 & 4.2060 & 7.6079 \\
\hline & 2 & 3.8378 & 6.9110 \\
\hline & 5 & 3.6539 & 6.1810 \\
\hline & 10 & 3.5459 & 5.7716 \\
\hline
\end{tabular}

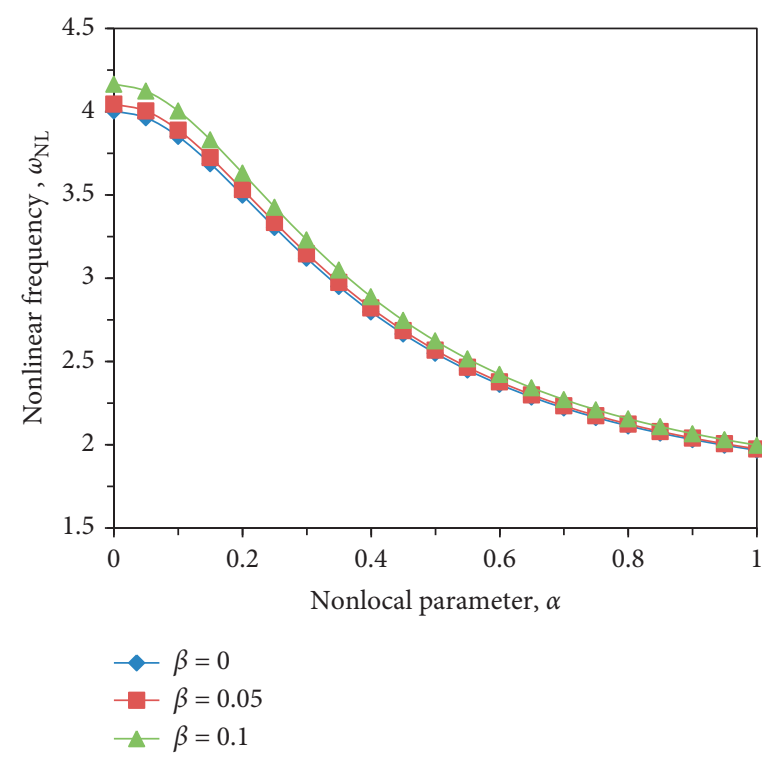

(a)

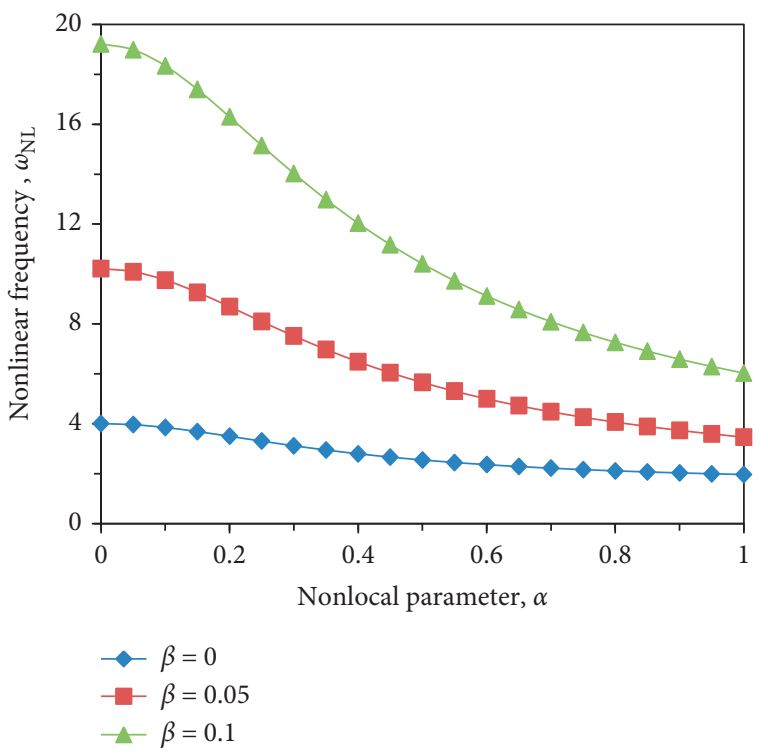

(b)

FIgURE 2: The variation of the nonlinear frequencies of the FG nanobeams to the nonlocal parameter for $k=5, L / h=15$, and $V_{0}=0.01$ : (a) without thickness effect; (b) with thickness effect. 


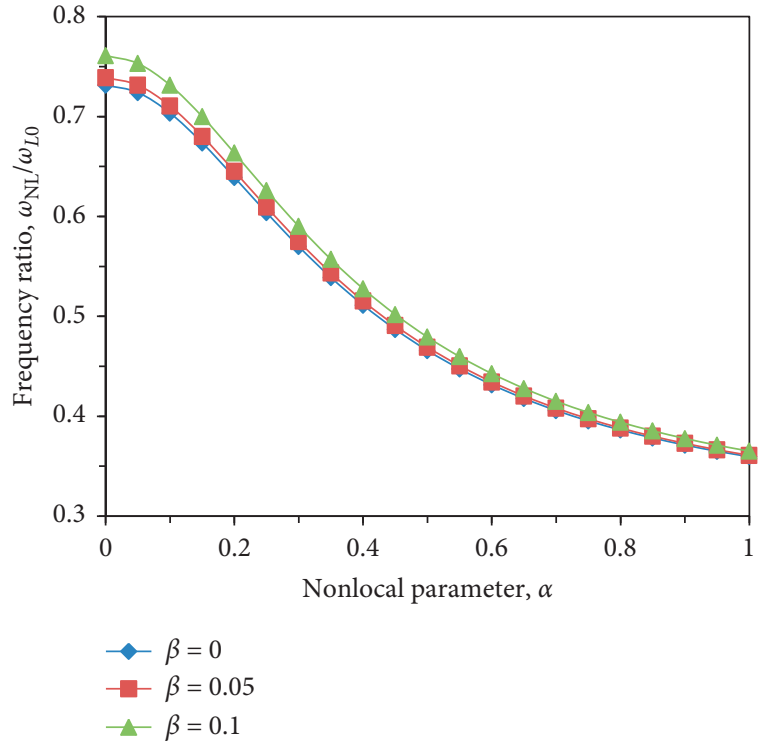

(a)

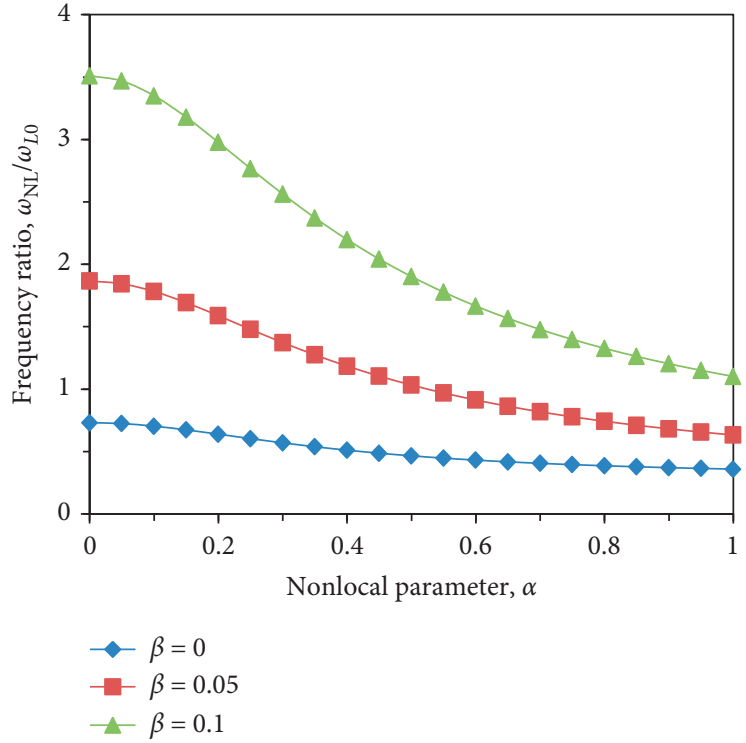

(b)

FIGURE 3: The variation of the frequency ratios of the FG nanobeams to the nonlocal parameter for $k=5, L / h=15$, and $V_{0}=0.01$ : (a) without thickness effect; (b) with thickness effect.

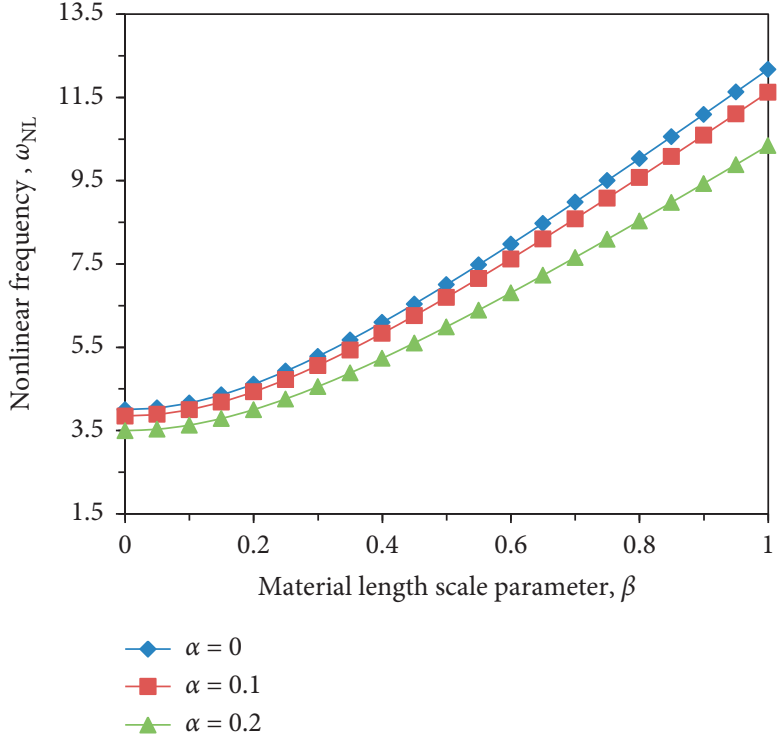

(a)

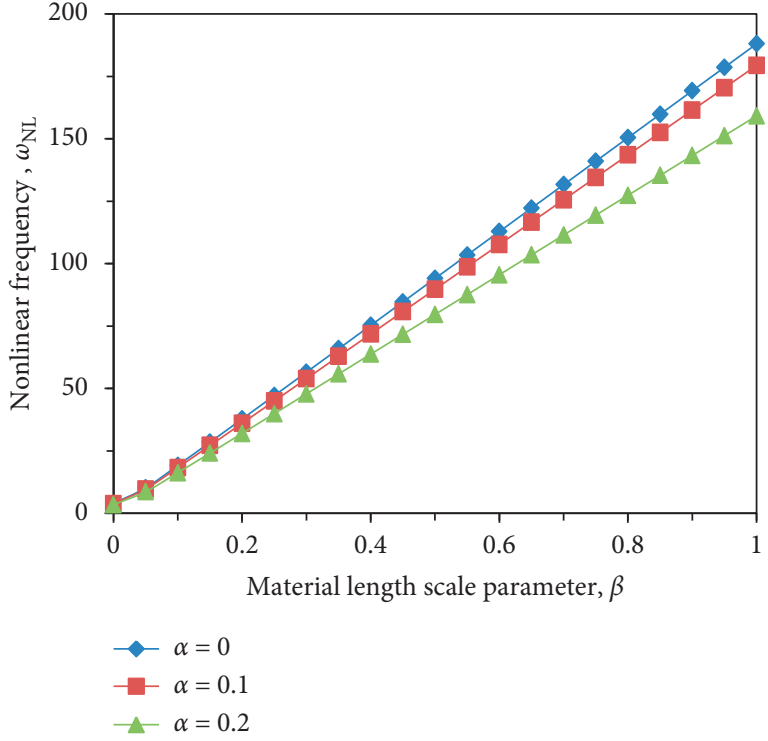

(b)

Figure 4: The variation of the nonlinear frequencies of the FG nanobeams to the material length scale parameter for $k=5, L / h=15$, and $V_{0}=0.01$ : (a) without thickness effect; (b) with thickness effect.

power-law index $k$ decreases the natural frequency of the FG nanobeam. With each specific value of the power-law index and the slenderness ratio, the natural frequency of the FG nanobeam considering the thickness effect is always greater than the natural frequency of the FG nanobeam without considering the thickness effect.

To investigate effects of the material length scale parameter $\beta=l_{m} / L$ and the nonlocal parameter $\alpha=e a / L$ on the vibration responses of the FG nanobeam, we introduce the scale ratio $c$ and the frequency ratio $\omega_{\text {ratio }}$, respectively, which are defined as

$$
\begin{aligned}
c & =\frac{\alpha}{\beta}=\frac{e a}{l_{m}}, \\
\omega_{\text {ratio }} & =\frac{\omega_{\mathrm{NL}}}{\omega_{L 0}},
\end{aligned}
$$




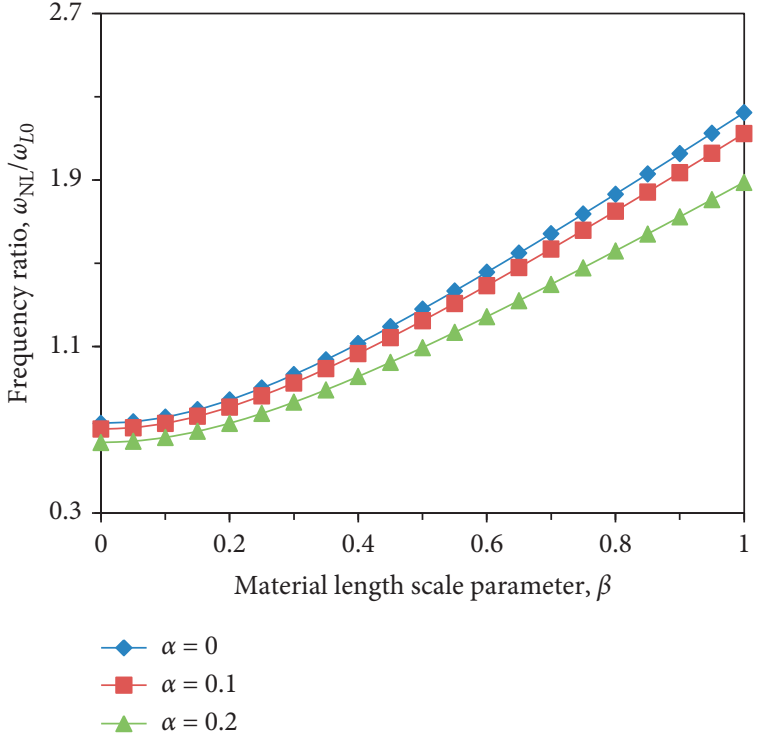

(a)

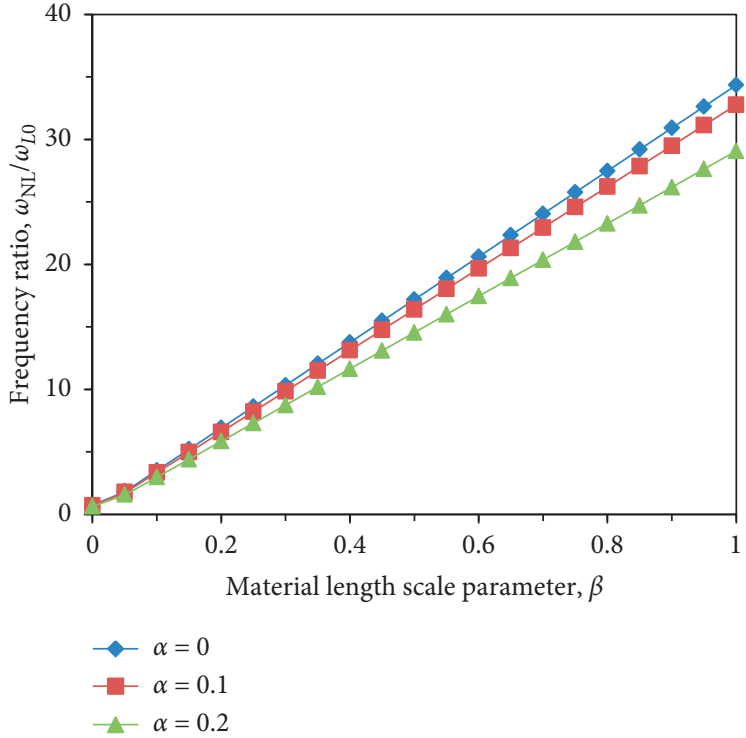

(b)

FIgURE 5: The variation of the frequency ratios of the FG nanobeams to the material length scale parameter for $k=5, L / h=15$, and $V_{0}=0.01$ : (a) without thickness effect; (b) with thickness effect.

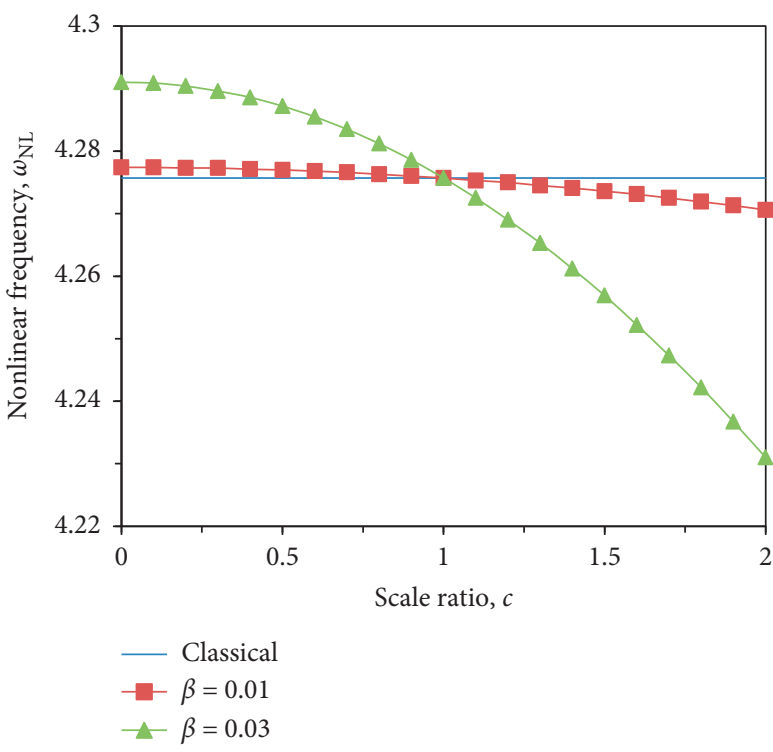

(a)

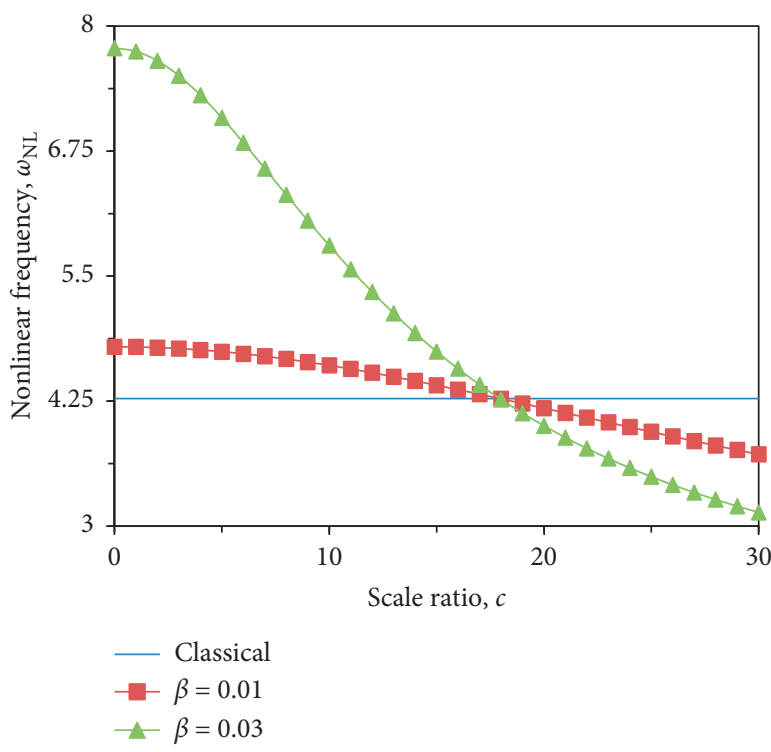

(b)

FIGURE 6: The variation of the nonlinear frequencies of the FG nanobeams to the scale ratio for $k=2, L / h=15$, and $V_{0}=0.01$ : (a) without thickness effect; (b) with thickness effect.

where $\omega_{L 0}$ is the linear frequency of the homogeneous beam using the classical elasticity theory.

Effects of the material length scale parameter $\left(\beta=l_{m} / L\right)$ and the nonlocal parameter $(\alpha=e a / L)$ on the nonlinear vibration response of the FG nanobeam are presented in Figures 2-7. Figures 2-5 show the variations of the nonlinear frequencies and the frequency ratios of the FG nanobeams to the nonlocal parameter and the material length scale parameter. It can be concluded that both nonlinear frequency and frequency ratio of the nanobeam decrease as the nonlocal parameter increases and increase as the material length scale parameter increases.

The effects of both nonlocal and material length scale parameters are integrated in the NSGT; thus, to have a better observation of the effects of these two parameters on the vibration response of the nanobeam, variations of the 


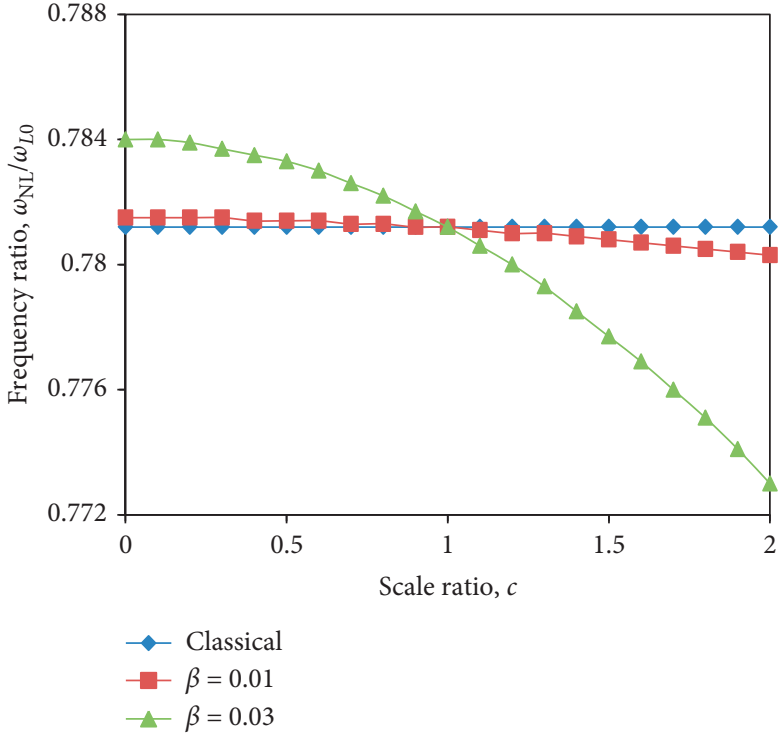

(a)

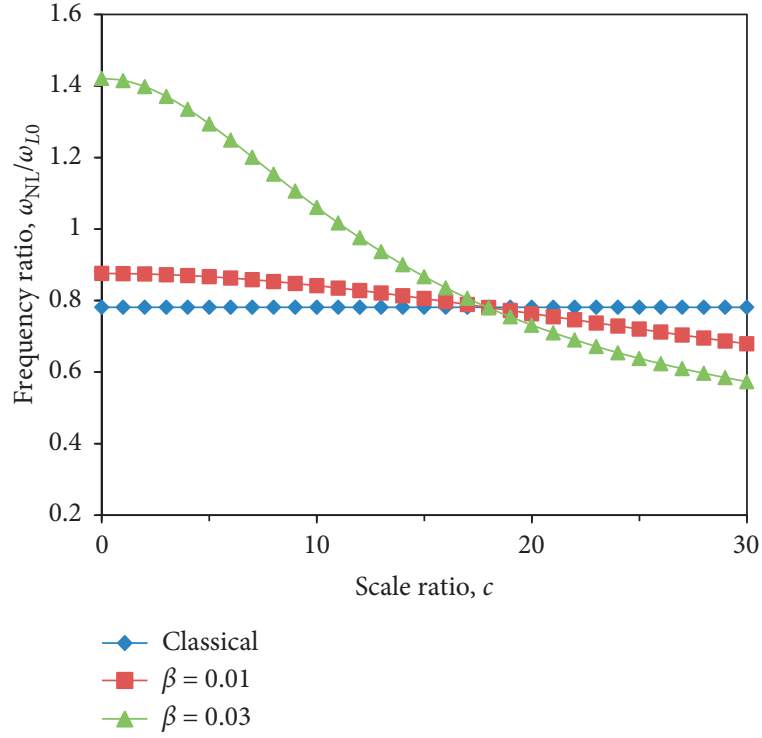

(b)

Figure 7: The variation of the frequency ratios of the FG nanobeams to the scale ratio for $k=2, L / h=15$, and $V_{0}=0.01$; (a) without thickness effect; (b) with thickness effect.

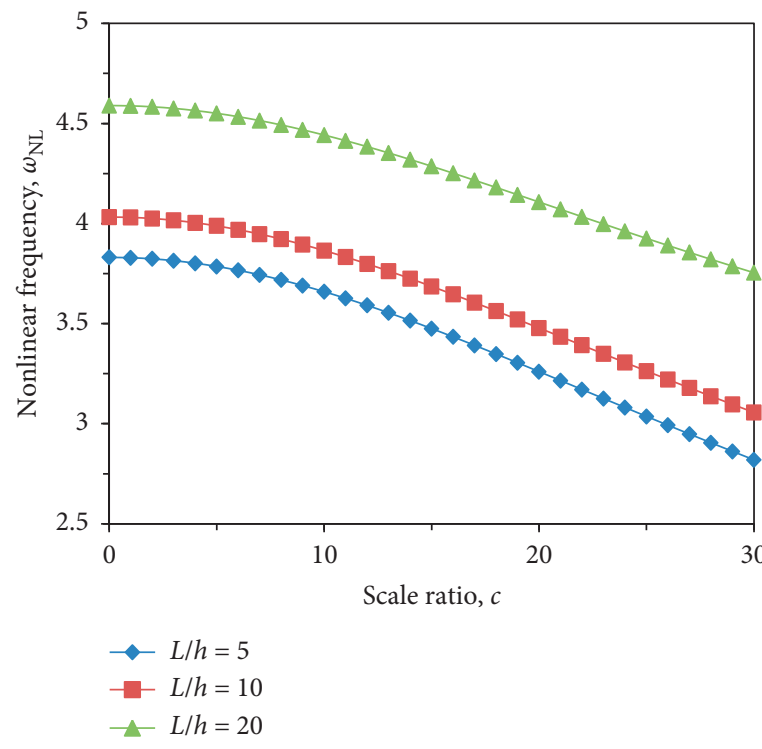

(a)

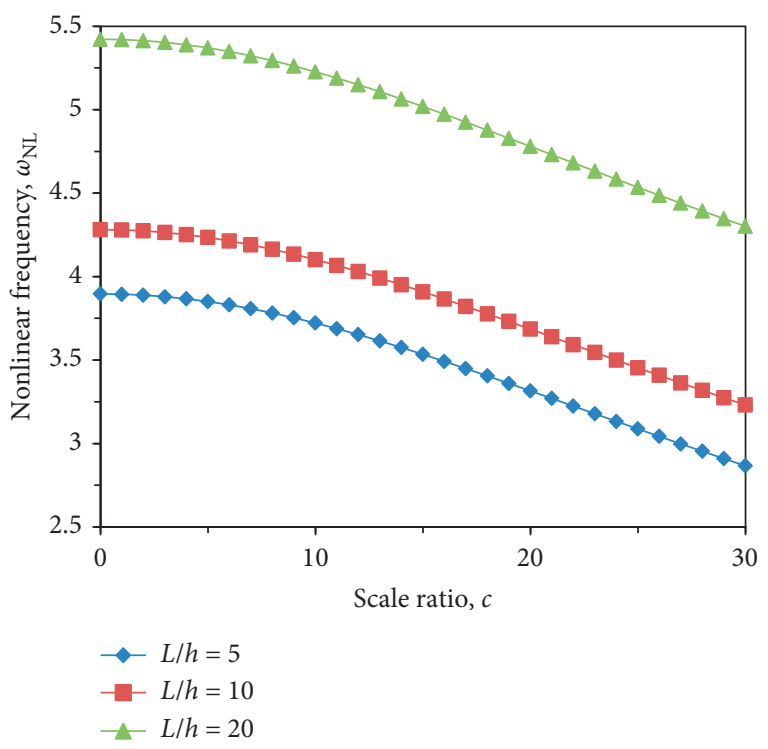

(b)

FIGURE 8: The variation of the nonlinear frequencies of the FG nanobeams to the scale ratio for $k=2, \beta=0.01$, and $V_{0}=0.01$ : (a) without thickness effect; (b) with thickness effect.

nonlinear frequencies and frequency ratios of the nanobeam to the scale ratio $c$ are investigated. Figures 6 and 7 show the variations of the nonlinear frequencies and the frequency ratios of the FG nanobeams, respectively, to the scale ratio $c$ for some different values of the material length scale parameter. As expected, when the thickness effect is not considered (Figures 6(a) and 7(a)), when $c=1$ (i.e., $e a=l_{m}$ ), the nonlinear frequencies and frequency ratios of the FG classical beams are equal to the ones of the FG nonlocal strain gradient beams; the FG nanobeam shows a stiffnesssoftening effect if $c>1$ (i.e., $e a>l_{m}$ ) and shows a stiffnesshardening effect if $c<1$ (i.e., $e a<l_{m}$ ). These results completely agree with the results obtained by $\mathrm{Li}$ and $\mathrm{Hu}$ [52]. As in Figures 6(a) and 7(a), without considering the thickness effect, the nonlinear frequencies and frequency ratios of the FG nanobeams increase as the material length scale parameter $\left(\beta=l_{m} / L\right)$ increases if $c<1$, and the nonlinear frequencies and frequency ratios of the FG nanobeams 


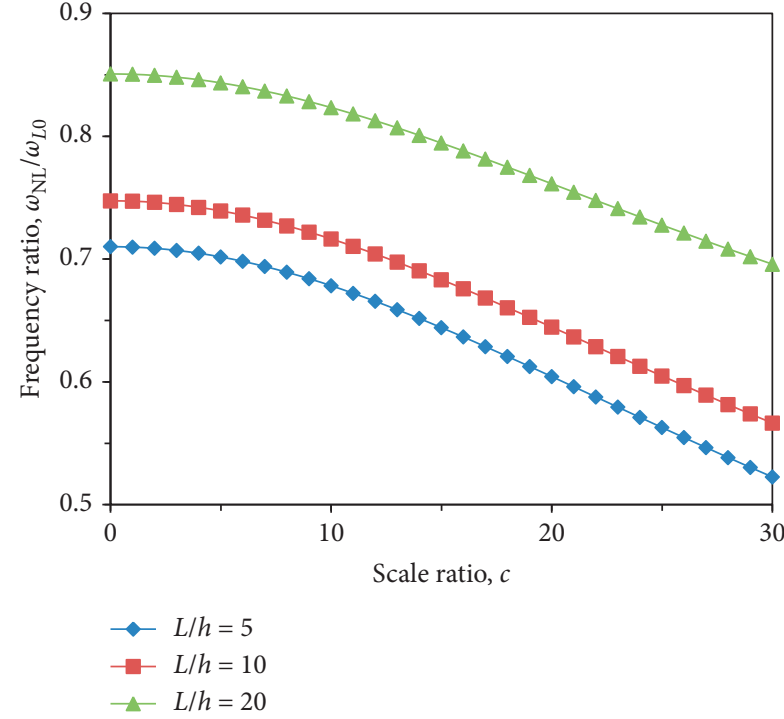

(a)

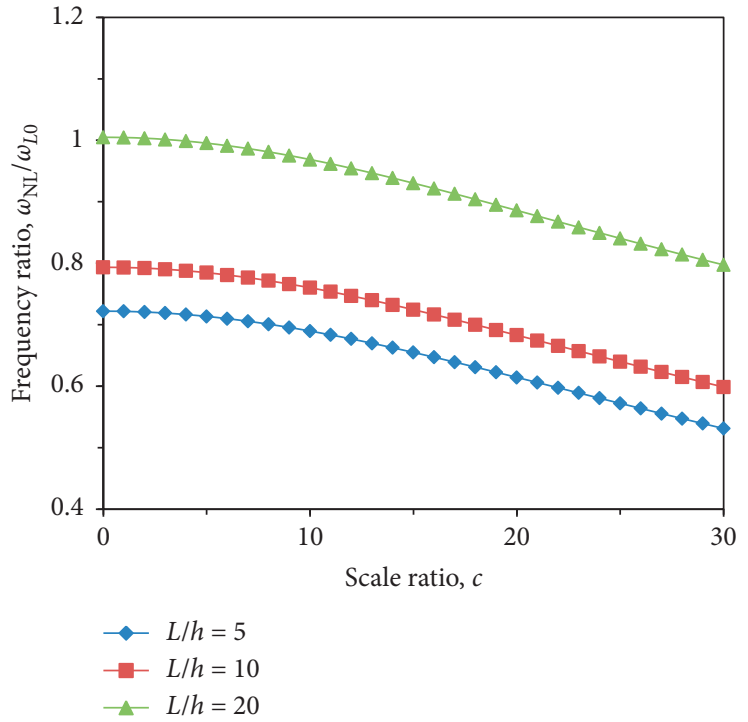

(b)

FIgURE 9: The variation of the frequency ratios of the FG nanobeams to the scale ratio for $k=2, \beta=0.01$, and $V_{0}=0.01$ : (a) without thickness effect; (b) with thickness effect.

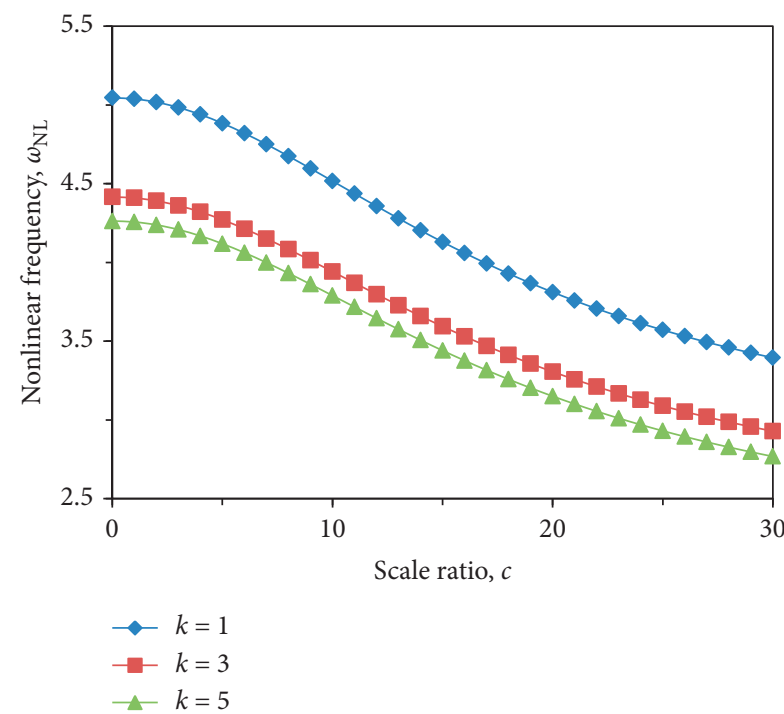

(a)

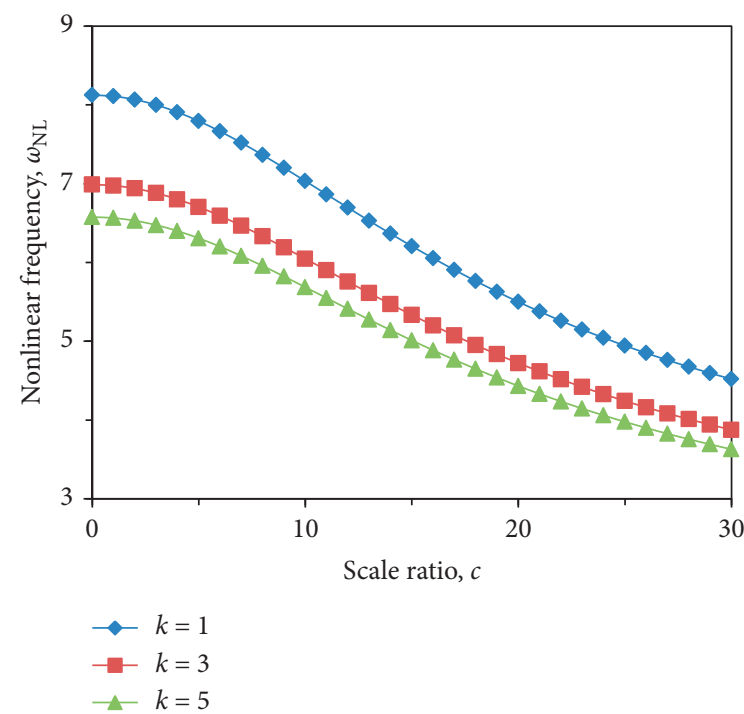

(b)

FIGURE 10: The variation of the nonlinear frequencies of the FG nanobeams to the scale ratio for $\beta=0.02, L / h=20$, and $V_{0}=0.01:(a)$ without thickness effect; (b) with thickness effect.

decrease as the material length scale parameter $\left(\beta=l_{m} / L\right)$ increases if $c>1$. However, when the thickness effect is considered as shown in Figures 6(b) and 7(b), we see that the slenderness ratio $L / h$ has a great influence on the size-dependent vibration response of the FG nanobeam. From Figures 6(b) and 7(b), it can be observed that, for a selected specific value of the power-law index $k=2$, the slenderness ratio $L / h=15$ and the initial amplitude $V_{0}=0.01$. The FG nanobeam shows a stiffness-softening effect if $c>18$ (i.e., $e a>18 l_{m}$ ) and a stiffness-hardening effect if $c<18$ (i.e., $e a<18 l_{m}$ ); the nonlinear frequencies and frequency ratios of the FG nonlocal strain gradient beams are equal to the ones of the FG classical beams if $e a=18 l_{m}$. From Figure 7, we see that the frequency ratio of the FG nanobeam considering the thickness effect is always larger than the frequency ratio of the FG nanobeam without considering the thickness effect. The frequency ratio of the FG nanobeam decreases when the scale ratio increases.

Effect of the slenderness ratio $L / h$ on the nonlinear vibration response of the FG nanobeams is also investigated 


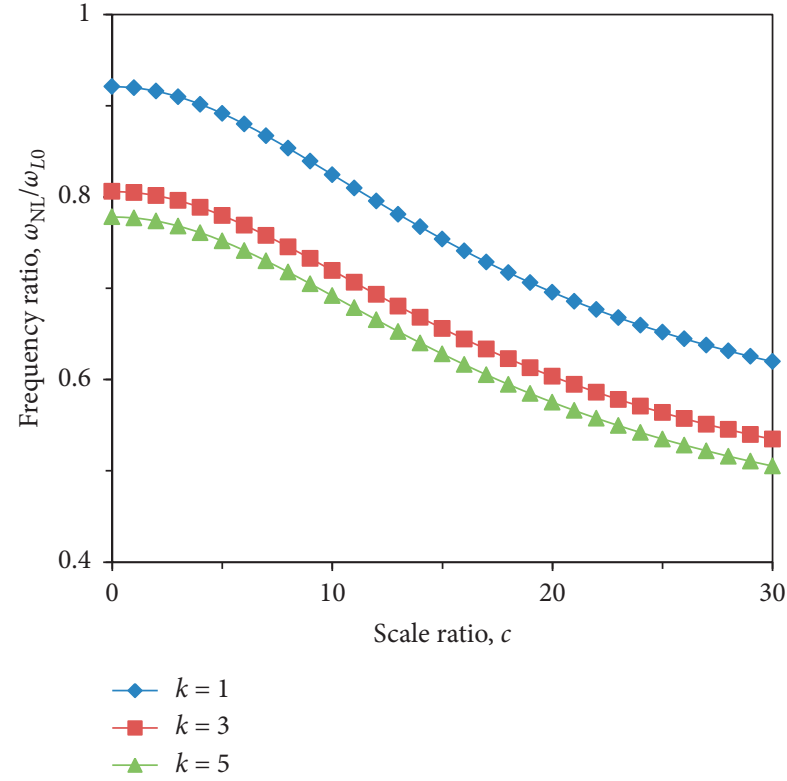

(a)

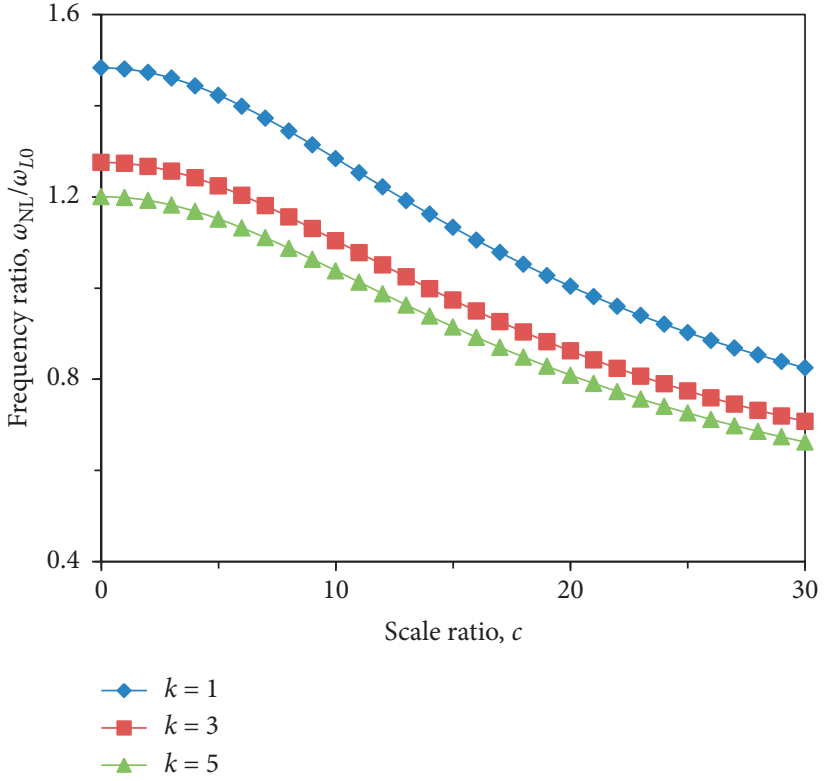

(b)

FIgURE 11: The variation of the frequency ratios of the FG nanobeams to the scale ratio for $\beta=0.02, L / h=20$, and $V_{0}=0.01$ : (a) without thickness effect; (b) with thickness effect.

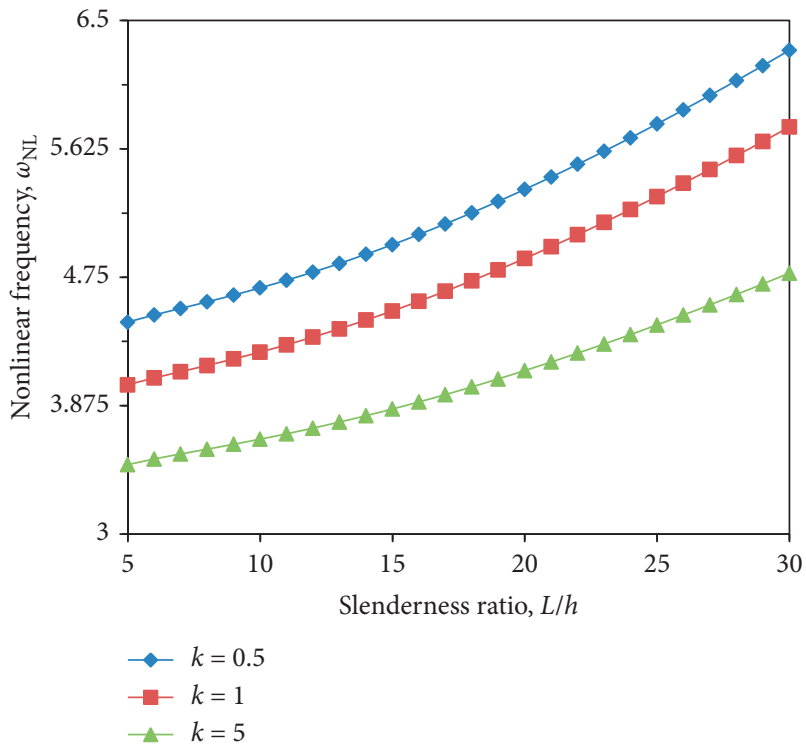

(a)

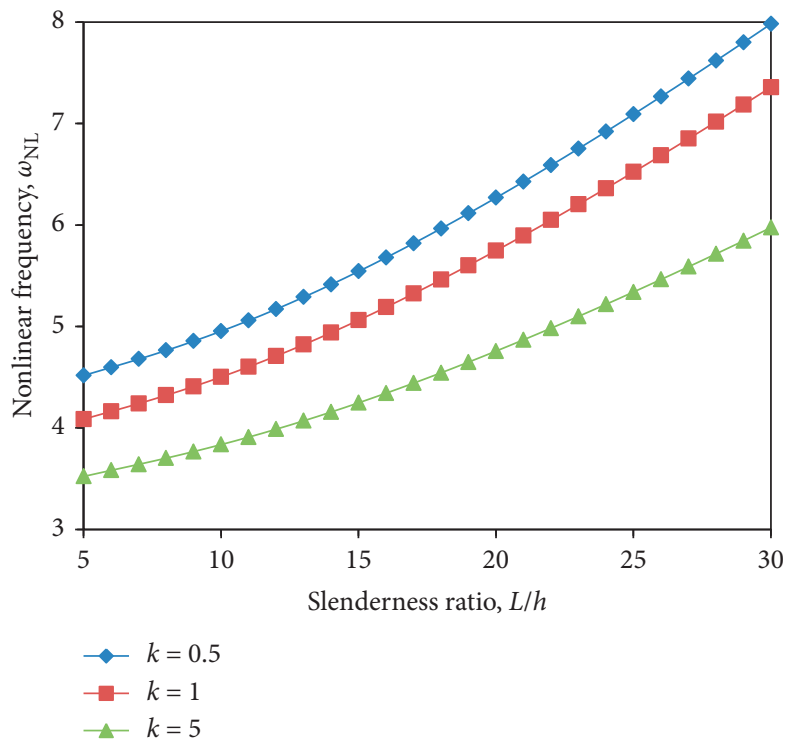

(b)

FIGURE 12: The variation of the nonlinear frequencies of the FG nanobeams to the slenderness ratio for $\beta=0.01, c=10$, and $V_{0}=0.01$ : (a) without thickness effect; (b) with thickness effect.

and shown in Figures 8 and 9. These figures show the variations of the nonlinear frequencies and frequency ratios of the FG nanobeams to the scale ratio $c$ for some differential values of the slenderness ratio $L / h$. It can be concluded that the slenderness ratio $L / h$ leads to an increase in the nonlinear frequencies and the frequency ratios of the FG nanobeams.
However, with the specific value of the slenderness ratio, both nonlinear frequencies and frequency ratios of the FG nanobeams decrease when the scale ratio increases.

Finally, the effect of the power-law index $k$ on the nonlinear vibration response of the FG nanobeams is examined. Figures 10 and 11 show the variations of the 


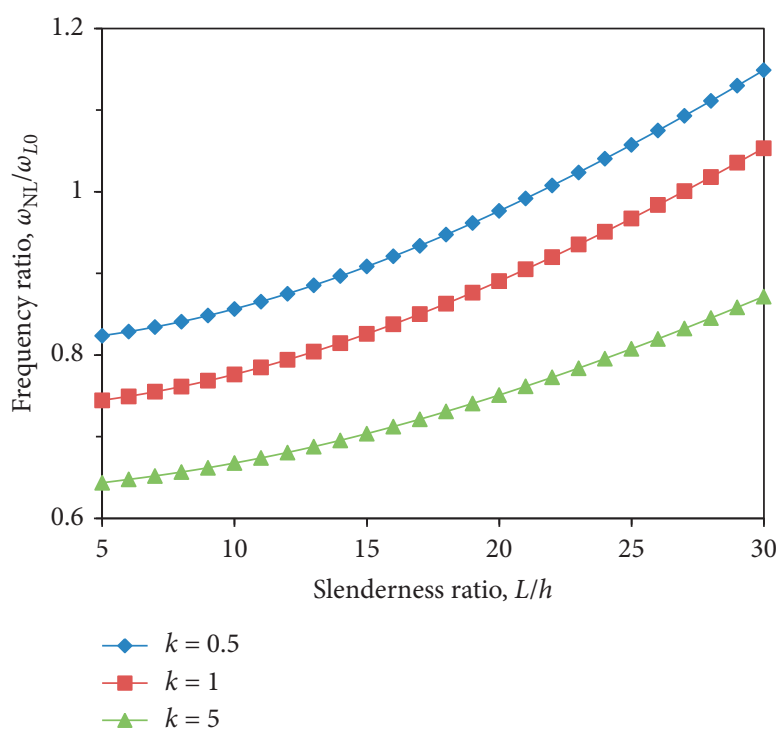

(a)

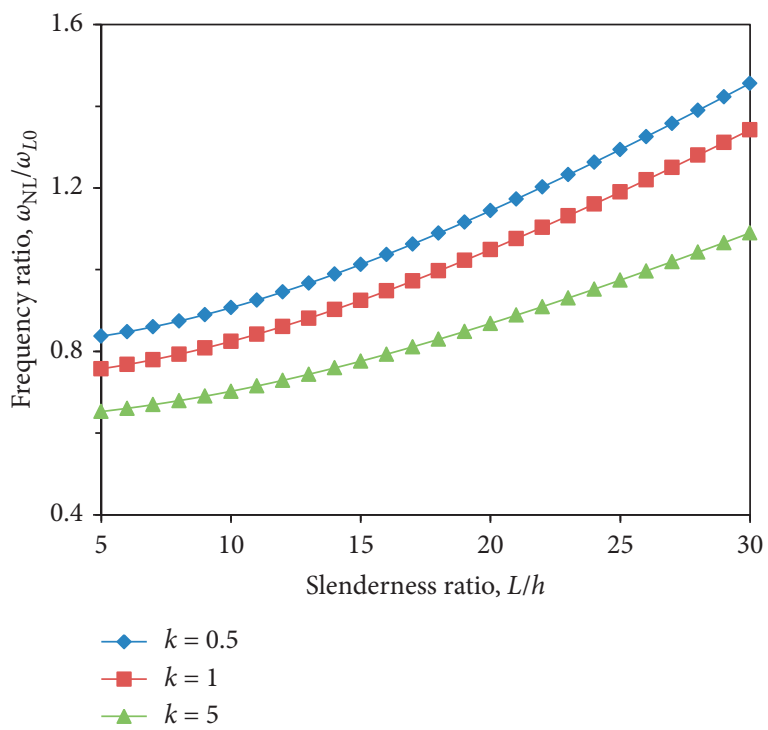

(b)

FIGURE 13: The variation of the frequency ratios of the FG nanobeams to the slenderness ratio for $\beta=0.01, c=10$, and $V_{0}=0.01$ : (a) without thickness effect; (b) with thickness effect.

nonlinear frequencies and frequency ratios of the FG nanobeams to the scale ratio $c$ for some various values of the power-law index $k$, respectively, while Figures 12 and 13 show the variations of the nonlinear frequencies and frequency ratios of the FG nanobeams to the slenderness ratio $L / h$ for some various values of the power-law index $k$, respectively. It can be observed that both nonlinear frequencies and the frequency ratios of the FG nanobeams decrease when the power-law index $k$ increases. However, for each specific value of the power-law index $k$, the nonlinear frequencies and the frequency ratios of the FG nanobeam decrease as the scale ratio $c$ increases and increase as the slenderness ratio $L / h$ increases. The nonlinear frequencies of the FG nanobeam considering the thickness effect are always larger than the ones without considering the thickness effect.

\section{Conclusion}

A nonlocal strain gradient Euler-Bernoulli beam model considering the effect of the thickness is developed to analyze the size-dependent vibration response of the functionally graded nanobeam. With the help of Galerkin's technique and the Hamiltonian approach, the amplitude-frequency relationship of the pinned-pinned FG nanobeam is obtained in a closed form.

When considering the thickness effect, the size-dependent vibration response of the nanobeam depends not only on the scale ratio $c=e a / l_{m}$ (the ratio of nonlocal parameter ea to material length scale parameter $l_{m}$ ) but also on the slenderness ratio $L / h$. The thickness affects greatly the sizedependent vibration response of the FG nanobeam.

Effects of the slenderness ratio $L / h$ and the power-law index $k$ on the nonlinear vibration response of the FG nanobeam are also investigated. It is found that the slenderness ratio leads to an increase in the linear and nonlinear frequencies of the FG nanobeams, while the power-law index $k$ leads to a decrease in the linear and nonlinear frequencies of the FG nanobeams.

When the thickness effect is not considered, the nonlinear frequencies and frequency ratios of the FG nanobeams increase as the material length scale parameter $\left(\beta=l_{m} / L\right)$ increases if $e a<l_{m}$, and the nonlinear frequencies and frequency ratios of the FG nanobeams decrease as the material length scale parameter $\left(\beta=l_{m} / L\right)$ increases if $e a>l_{m}$. However, when the thickness effect is considered, the slenderness ratio $L / h$ has a great influence on the size-dependent vibration response of the FG nanobeam. For a selected specific value of the power-law index $k=2$, the slenderness ratio $L / h=15$ and the initial amplitude $V_{0}=0.01$. The FG nanobeam shows a stiffness-softening effect if $c>18$ (i.e., $e a>18 l_{m}$ ) and a stiffness-hardening effect if $c<18$ (i.e., $e a<18 l_{m}$ ); the nonlinear frequencies and frequency ratios of the FG nonlocal strain gradient beams are equal to the ones of the FG classical beams if $e a=18 l_{m}$.

\section{Data Availability}

The data used to support the findings of this study are available from the corresponding author upon request.

\section{Conflicts of Interest}

The authors declare that they have no conflicts of interest.

\section{Acknowledgments}

This research was funded by the Vietnam National Foundation for Science and Technology Development (NAFOSTED) under grant no. 107.01-2018.304. 


\section{References}

[1] N. A. Fleck, G. M. Muller, M. F. Ashby, and J. W. Hutchinson, "Strain gradient plasticity: theory and experiment," Acta Metallurgica et Materialia, vol. 42, no. 2, pp. 475-487, 1994.

[2] A. C. M. Chong and D. C. C. Lam, "Strain gradient plasticity effect in indentation hardness of polymers," Journal of Materials Research, vol. 14, no. 10, pp. 4103-4110, 1999.

[3] A. C. Eringen and D. G. B. Edelen, "On nonlocal elasticity," International Journal of Engineering Science, vol. 10, no. 3, pp. 233-248, 1972.

[4] A. C. Eringen, "On differential equations of nonlocal elasticity and solutions of screw dislocation and surface waves," Journal of Applied Physics, vol. 54, no. 9, pp. 4703-4710, 1983.

[5] J. N. Reddy, "Nonlocal theories for bending, buckling and vibration of beams," International Journal of Engineering Science, vol. 45, no. 2-8, pp. 288-307, 2007.

[6] M. Aydogdu, "A general nonlocal beam theory: its application to nanobeam bending, buckling and vibration," Physica E: Low-Dimensional Systems and Nanostructures, vol. 41, no. 9, pp. 1651-1655, 2009.

[7] C. M. Wang, S. Kitipornchai, C. W. Lim, and M. Eisenberger, "Beam bending solutions based on nonlocal Timoshenko beam theory," Journal of Engineering Mechanics, vol. 134, no. 6, pp. 475-481, 2008.

[8] Q. Wang and K. M. Liew, "Application of nonlocal continuum mechanics to static analysis of micro- and nano-structures," Physics Letters A, vol. 363, no. 3, pp. 236-242, 2007.

[9] C. M. Wang, Y. Y. Zhang, and X. Q. He, "Vibration of nonlocal Timoshenko beams," Nanotechnology, vol. 18, no. 10, Article ID 105401, 2007.

[10] H.-T. Thai, "A nonlocal beam theory for bending, buckling, and vibration of nanobeams," International Journal of Engineering Science, vol. 52, pp. 56-64, 2012.

[11] M. A. Eltaher, M. E. Khater, and S. A. Emam, "A review on nonlocal elastic models for bending, buckling, vibrations, and wave propagation of nanoscale beams," Applied Mathematical Modelling, vol. 40, no. 5-6, pp. 4109-4128, 2016.

[12] M. Aydogdu, "Vibration of multi-walled carbon nanotubes by generalized shear deformation theory," International Journal of Mechanical Sciences, vol. 50, no. 4, pp. 837-844, 2008.

[13] J. N. Reddy and S. D. Pang, "Nonlocal continuum theories of beams for the analysis of carbon nanotubes," Journal of Applied Physics, vol. 103, no. 2, Article ID 023511, 2008.

[14] A. Benzair, A. Tounsi, A. Besseghier, H. Heireche, N. Moulay, and L. Boumia, "The thermal effect on vibration of singlewalled carbon nanotubes using nonlocal Timoshenko beam theory," Journal of Physics D: Applied Physics, vol. 41, no. 22, Article ID 225404, 2008.

[15] D. Kumar, C. Heinrich, and A. M. Waas, "Buckling analysis of carbon nanotubes modeled using nonlocal continuum theories," Journal of Applied Physics, vol. 103, no. 7, Article ID 073521, 2008.

[16] T. Murmu and S. C. Pradhan, "Buckling analysis of a singlewalled carbon nanotube embedded in an elastic medium based on nonlocal elasticity and Timoshenko beam theory and using DQM," Physica E: Low-Dimensional Systems and Nanostructures, vol. 41, no. 7, pp. 1232-1239, 2009.

[17] T. Murmu and S. C. Pradhan, "Thermo-mechanical vibration of a single-walled carbon nanotube embedded in an elastic medium based on nonlocal elasticity theory," Computational Materials Science, vol. 46, no. 4, pp. 854-859, 2009.

[18] Y. Q. Zhang, G. R. Liu, and X. Y. Xie, "Free transverse vibrations of double-walled carbon nanotubes using a theory of nonlocal elasticity," Physics Reviews B, vol. 71, no. 19, Article ID 195404, 2005.

[19] Y. Y. Zhang, C. M. Wang, W. H. Duan, Y. Xiang, and Z. Zong, "Assessment of continuum mechanics models in predicting buckling strains of single-walled carbon nanotubes," Nanotechnology, vol. 20, no. 39, Article ID 395707, 2009.

[20] B. Arash and Q. Wang, "A review on the application of nonlocal elastic models in modeling of carbon nanotubes and graphenes," Computational Materials Science, vol. 51, no. 1, pp. 303-313, 2012.

[21] R. D. Mindlin and H. F. Tiersten, "Effects of couple-stresses in linear elasticity," Archive for Rational Mechanics and Analysis, vol. 11, no. 1, pp. 415-448, 1962.

[22] R. D. Mindlin, "Micro-structure in linear elasticity," Archive for Rational Mechanics and Analysis, vol. 16, no. 1, pp. 51-78, 1964.

[23] R. D. Mindlin, "Second gradient of strain and surface-tension in linear elasticity," International Journal of Solids and Structures, vol. 1, no. 1, pp. 417-438, 1965.

[24] R. A. Toupin, "Elastic materials with couple-stresses," Archive for Rational Mechanics and Analysis, vol. 11, no. 1, pp. 385414, 1962.

[25] E. C. Aifantis, "On the role of gradients in the localization of deformation and fracture," International Journal of Engineering Science, vol. 30, no. 10, pp. 1279-1299, 1992.

[26] F. Yang, A. C. M. Chong, D. C. C. Lam, and P. Tong, "Couple stress based strain gradient theory for elasticity," International Journal of Solids and Structures, vol. 39, no. 10, pp. 2731-2743, 2002.

[27] D. C. C. Lam, F. Yang, A. C. M. Chong, J. Wang, and P. Tong, "Experiments and theory in strain gradient elasticity," Journal of the Mechanics and Physics of Solids, vol. 51, no. 8, pp. 1477-1508, 2003.

[28] B. Wang, J. Zhao, and S. Zhou, "A micro scale Timoshenko beam model based on strain gradient elasticity theory," $E$ ropean Journal of Mechanics-A/Solids, vol. 29, no. 4, pp. 591-599, 2010.

[29] S. L. Kong, S. J. Zhou, Z. F. Nie, and K. Wang, "Static and dynamic analysis of micro beams based on strain gradient elasticity theory," International Journal of Engineering Science, vol. 47, pp. 487-498, 2008.

[30] H. Ma, X. Gao, and J. Reddy, "A microstructure-dependent Timoshenko beam model based on a modified couple stress theory," Journal of the Mechanics and Physics of Solids, vol. 56, no. 12 , pp. 3379-3391, 2008.

[31] S. K. Park and X.-L. Gao, "Bernoulli-Euler beam model based on a modified couple stress theory," Journal of Micromechanics and Microengineering, vol. 16, no. 11, pp. 23552359, 2006.

[32] B. Akgöz and Ö. Civalek, "Analysis of micro-sized beams for various boundary conditions based on the strain gradient elasticity theory," Archive of Applied Mechanics, vol. 82, no. 3, pp. 423-443, 2012.

[33] B. Akgöz and Ö. Civalek, "A size-dependent shear deformation beam model based on the strain gradient elasticity theory," International Journal of Engineering Science, vol. 70, pp. 1-14, 2013.

[34] C. M. C. Roque, D. S. Fidalgo, A. J. M. Ferreira, and J. N. Reddy, "A study of a microstructure-dependent composite laminated Timoshenko beam using a modified couple stress theory and a meshless method," Composite Structures, vol. 96, pp. 532-537, 2013.

[35] W. Chen, C. Weiwei, and K. Y. Sze, "A model of composite laminated Reddy beam based on a modified couple-stress theory," Composite Structures, vol. 94, pp. 2599-2609, 2012. 
[36] M. H. Ghayes, M. Amabili, and H. Farokhi, "Nonlinear forced vibrations of a microbeam based on the strain gradient elasticity theory," International Journal of Engineering Science, vol. 63, pp. 52-60, 2013.

[37] M. H. Ghayes, H. Farokhi, and M. Amabili, "Nonlinear dynamics of a microscale beam based on the modified couple stress theory," Composites Part B: Engineering, vol. 50, pp. 318-324, 2013.

[38] C. W. Lim, G. Zhang, and J. N. Reddy, "A higher-order nonlocal elasticity and strain gradient theory and its applications in wave propagation," Journal of the Mechanics and Physics of Solids, vol. 78, pp. 298-313, 2015.

[39] L. Li and Y. Hu, "Buckling analysis of size-dependent nonlinear beams based on a nonlocal strain gradient theory," International Journal of Engineering Science, vol. 97, pp. 8494, 2015.

[40] L. Lu, X. Guo, and J. Zhao, "Size-dependent vibration analysis of nanobeams based on the nonlocal strain gradient theory," International Journal of Engineering Science, vol. 116, pp. 12-24, 2017.

[41] L. Li, Y. Hu, X. Li, and L. Ling, "Size-dependent effects on critical flow velocity of fluid-conveying microtubes via nonlocal strain gradient theory," Microfluid Nanofluid, vol. 20, p. 76, 2016.

[42] R. Bahaadini, A. R. Saidi, A. R. Saidi, and M. Hosseini, "On dynamics of nanotubes conveying nanoflow," International Journal of Engineering Science, vol. 123, pp. 181-196, 2018.

[43] M. H. Ghayesh and H. Farokhi, "On the viscoelastic dynamics of fluid-conveying microtubes," International Journal of Engineering Science, vol. 127, pp. 186-200, 2018.

[44] M. Arefi, M. Kiani, and T. Rabczuk, "Application of nonlocal strain gradient theory to size dependent bending analysis of a sandwich porous nanoplate integrated with piezomagnetic face-sheets," Composites Part B: Engineering, vol. 168, pp. 320-333, 2019.

[45] M. Arefi, M. Kiani, and M. H. Zamani, "Nonlocal strain gradient theory for the magnetoelectro-elastic vibration response of a porous FG-core sandwich nanoplate with piezomagnetic face sheets resting on an elastic foundation," Journal of Sandwich Structures \& Materials, 2018.

[46] D. K. Jha, T. Kant, and R. K. Singh, "A critical review of recent research on functionally graded plates," Composite Structures, vol. 96, pp. 833-849, 2013.

[47] R. Ansari, R. Gholami, and S. Sahmani, "Free vibration analysis of size-dependent functionally graded microbeams based on the strain gradient Timoshenko beam theory," Composite Structures, vol. 94, no. 1, pp. 221-228, 2011.

[48] A. Arbind and J. N. Reddy, "Nonlinear analysis of functionally graded microstructure-dependent beams," Composite Structures, vol. 98, pp. 272-281, 2013.

[49] A. Fallah and M. M. Aghdam, "Nonlinear free vibration and post-buckling analysis of functionally graded beams on nonlinear elastic foundation," European Journal of MechanicsA/Solids, vol. 30, no. 4, pp. 571-583, 2011.

[50] H.-T. Thai and T. P. Vo, "Bending and free vibration of functionally graded beams using various higher-order shear deformation beam theories," International Journal of $\mathrm{Me}$ chanical Sciences, vol. 62, pp. 57-66, 2012.

[51] M. Simsek, "Nonlinear free vibration of a functionally graded nanobeam using nonlocal strain gradient theory and a novel Hamiltonian approach," International Journal of Engineering Science, vol. 105, pp. 12-27, 2016.

[52] L. Li and Y. Hu, "Nonlinear bending and free vibration analyses of nonlocal strain gradient beams made of functionally graded material," International Journal of Engineering Science, vol. 107, pp. 77-97, 2016.

[53] M. N. M. Allam and A. F. Radwan, "Nonlocal strain gradient theory for bending, buckling, and vibration of viscoelastic functionally graded curved nanobeam embedded in an elastic medium," Advances in Mechanical Engineering, vol. 11, no. 4, pp. 1-15, 2019.

[54] S. Esfahani, S. Esmaeilzade Khadem, A. Ebrahimi Mamaghani, and A. E. Mamaghani, "Nonlinear vibration analysis of an electrostatic functionally graded nano-resonator with surface effects based on nonlocal strain gradient theory," International Journal of Mechanical Sciences, vol. 151, pp. 508-522, 2019.

[55] M. Arefi, E. M.-R. Bidgoli, E. Mohammad-Rezaei Bidgoli, R. Dimitri, M. Bacciocchi, and F. Tornabene, "Application of sinusoidal shear deformation theory and physical neutral surface to analysis of functionally graded piezoelectric plate," Composites Part B: Engineering, vol. 151, pp. 35-50, 2018.

[56] M. Arefi, E. M.-R. Bidgoli, E. Mohammad-Rezaei Bidgoli, and A. M. Zenkour, "Free vibration analysis of a sandwich nanoplate including FG core and piezoelectric face-sheets by considering neutral surface," Mechanics of Advanced Materials and Structures, vol. 26, no. 9, pp. 741-752, 2019.

[57] L. Li, H. Tang, and Y. Hu, "The effect of thickness on the mechanics of nanobeams," International Journal of Engineering Science, vol. 123, pp. 81-91, 2018.

[58] W. Chen, L. Wang, and H.-L. Dai, "Nonlinear free vibration of nanobeams based on nonlocal strain gradient theory with the consideration of thickness-dependent size effect," Journal of Mechanics of Materials and Structures, vol. 14, no. 1, 2019.

[59] J.-H. He, "Hamiltonian approach to nonlinear oscillators," Physics Letters A, vol. 374, no. 23, pp. 2312-2314, 2010. 